\title{
Autopoiesis and Its Efficacy-A Metacybernetic View
}

\author{
Maurice Yolles ${ }^{1, *(D)}$ and B. Roy Frieden ${ }^{2}$ \\ 1 The Business School, Liverpool John Moores University, Liverpool L3 5UX, UK \\ 2 The Institute of Optics, University of Rochester, Rochester, NY 14627, USA; friedenr@optics.arizona.edu \\ * Correspondence: prof.m.yolles@gmail.com
}

\section{check for}

updates

Citation: Yolles, M.; Frieden, B.R. Autopoiesis and Its Efficacy-A

Metacybernetic View. Systems 2021, 9 ,

75. https://doi.org/10.3390/

systems 9040075

Academic Editor: Francis Heylighen

Received: 17 August 2021

Accepted: 22 October 2021

Published: 25 October 2021

Publisher's Note: MDPI stays neutral with regard to jurisdictional claims in published maps and institutional affiliations.

Copyright: (c) 2021 by the authors. Licensee MDPI, Basel, Switzerland. This article is an open access article distributed under the terms and conditions of the Creative Commons Attribution (CC BY) license (https:/ / creativecommons.org/licenses/by/ $4.0 /)$.

\begin{abstract}
This paper seeks to explain the nature of autopoiesis and its capacity to be efficacious, and to do this, it uses agency theory as embedded in metacybernetics. Agency, as a generalised intelligent adaptive living system, can anticipate the future once it has internalised a representation of an active contextual situation through autopoiesis. The role of observation and the nature of internalisation will be discussed, explaining that the latter has two states that determine agency properties of cognition. These are assimilation and accommodation. Assimilation is an information process and results in implicit cognition and recognition, whereas accommodation uses assimilated information delivering explicit cognition, recognition, and conscious awareness with rationality. Similarly, anticipation, a required property of the living, has two states, weak and strong, and these correspond to the two states of internalisation. Autopoiesis has various properties identifiable through the lenses of three autonomous but configurable schemas: General Collective Intelligence (GCI), Eigenform, and Extreme Physical Information (EPI). GCI is a pragmatic evolutionary approach concerned with a contextually connected purposeful and relatable set of task processes, each undertaken by a team of subagencies seeking collective fitness. Eigenform is a symbolic approach that is concerned with how observations can be suitably internalised and thus be used as a token to determine future behaviour, and how that which has been internalised can be adopted to anticipate the future. Extreme Physical Information (EPI) is an empirical approach concerned with acquiring information through observation of an unknown parameter through sampling regimes. The paper represents the conceptualisations of each schema in terms of autopoietic efficacy, and explores their configurative possibilities. It will adopt the ideas delivered to enhance explanations of the nature of autopoiesis and its efficacy within metacybernetics, providing a shift in thinking about autopoiesis and self-organisation.
\end{abstract}

Keywords: General Collective Intelligence; Extreme Physical Information; Eigenform; agency; metacybernetics; autopoiesis; efficacy

\section{Introduction}

This is a cybernetic paper concerned with living systems that use networks of autopoietic processes to enable them to have versatile adaptive behaviour, this enabling them to maintain their viability, i.e., to have a capacity to both survive and develop. Living systems are also cognitive systems [1], operating through information processes [2], and studied through cognitive science, which, for Simon [3], has a fundamental set of common concerns that are shared by the disciplines of psychology, computer science, linguistics, economics, epistemology, and the social sciences, generally all connected with information processing and adaptive systems. For Griffin [4], versatile adaptive behaviour provides evidence of consciousness. That living systems are conscious [5] is important because this allows one to differentiate between classes of living systems by distinguishing between degrees of consciousness, where more primitive forms of life are less complex with a low degree of consciousness, and more advanced forms are more complex with a higher degree of consciousness. Such a gradation has been proposed by Bitbol and Luisi [6,7], who offer a model of five evolutionary stages of system consciousness, these part of a hierarchical 
relationship. The different stages are each determined by the system's capability to internalise any environmental observations relevant to its life that it is capable of making, this then being used to determine its future-a process that may be expressed as anticipation. The hierarchic relationship is therefore defined in terms of the embeddedness of degrees of internalisation that it has access to. An alternative scheme comes from Bielecki [8], also offering a five-stage hierarchy of cognitive entities, each stage with its own cognitive abilities and functionalities, but this one comes from classifications that arise through considerations of perception and knowledge. Some broad synergy between the two might be expected since internalisation is dependent on perception that results in observations that then become internalised, and cognitive knowledge is a consequence of internalisation. Thus, one would expect the two schemas to have a similar trajectory of meaning. This is shown to be the case in Table 1.

Table 1. Relationship between the Bitbol and Luisi and Bielecki stages of living system development.

\begin{tabular}{|c|c|c|c|}
\hline $\begin{array}{l}\text { Consciousness } \\
\text { Stage }\end{array}$ & Bitbol and Luisi Hierarchy & Bielecki Hierarchy & Stage Relationship \\
\hline 1 & $\begin{array}{l}\text { Null pre-conscious. Devoid of } \\
\text { internalisation. }\end{array}$ & $\begin{array}{c}\text { Reflexive. Living system can only } \\
\text { create behaviours that directly } \\
\text { support existence and remove } \\
\text { threats. }\end{array}$ & $\begin{array}{l}\text { Null preconscious occurs prior to } \\
\text { reflexive since, in the former, threats } \\
\text { cannot be recognised. }\end{array}$ \\
\hline 2 & $\begin{array}{c}\text { Limited consciousness. Integration } \\
\text { of environmental factors. }\end{array}$ & $\begin{array}{l}\text { Associative. Able to undertake } \\
\text { simple analysis of direct } \\
\text { cause-and-effect relationships. }\end{array}$ & $\begin{array}{l}\text { Limited consciousness occurs at a } \\
\text { stage prior to associative, the former } \\
\text { being devoid of analytic ability. }\end{array}$ \\
\hline 3 & $\begin{array}{l}\text { Enduring modifications in } \\
\text { self-production. Stable dynamic } \\
\text { support provided able to deliver } \\
\text { strongly anticipative behaviour. }\end{array}$ & $\begin{array}{l}\text { Conscious. Can model complex } \\
\text { cause-and-effect chains, with a } \\
\text { conditional option permitting } \\
\text { future events variants, and an } \\
\text { ability for complex strategies of } \\
\text { activity. }\end{array}$ & $\begin{array}{l}\text { Enduring modifications in } \\
\text { self-production is approximated by } \\
\text { the consciousness stage since } \\
\text { cause-effect chains deliver strategy } \\
\text { that implies anticipation. }\end{array}$ \\
\hline 4 & $\begin{array}{l}\text { More complex changes that influence } \\
\text { behaviour. Involves observation of } \\
\text { the exterior, but without } \\
\text { awareness of an external } \\
\text { independent world. }\end{array}$ & $\begin{array}{l}\text { Self-consciousness. Epistemic } \\
\text { perspective can change, with } \\
\text { awareness of the existence of } \\
\text { conscious goals perhaps devoid of } \\
\text { proven reliable criteria. }\end{array}$ & $\begin{array}{l}\text { More complex changes are prior to } \\
\text { self-consciousness since the proof } \\
\text { requires awareness and access to } \\
\text { the outside independent world. }\end{array}$ \\
\hline 5 & $\begin{array}{l}\text { Collective consciousness that } \\
\text { recognises social aspects. } \\
\text { Knowledge develops by ascribing } \\
\text { properties to intersubjective } \\
\text { invariants. Intersubjectively } \\
\text { shared predictive common rules } \\
\text { become a collective consciousness } \\
\text { obeying internal closure }\end{array}$ & $\begin{array}{l}\text { The hypothetical omniscient stage, } \\
\text { with proven criteria and proof of } \\
\text { the reliability to use it. }\end{array}$ & $\begin{array}{l}\text { Collective consciousness is likely } \\
\text { equivalent to omniscient if one } \\
\text { considers that proof is a } \\
\text { social phenomenon. }\end{array}$ \\
\hline
\end{tabular}

Properties of cognition and consciousness are important to living systems, but there are underlying mechanisms that can explain the capacity to live. One conceptualisation of this comes from Korzeniewski [9], who considers that living systems are complex and cybernetic in nature. By complexity is meant the involvement of: (a) dissipative systems which increase their entropy (or uncertainty) as they move towards equilibrium, while using energy to create structured spots of locally decreasing entropy that are responsible for local order and organisation; and (b) a system hierarchy composed of subsystems that, in turn, have their own subsystems, and so on [10]. Cybernetics suggest control and communication [11], and this involves processes of feedback and feedforth. Now, Korzeniewski is concerned with biological living systems, and he limits his consideration of cybernetic processes to "inferior negative feedbacks" that are concerned with regulation, and their related "subordinate positive feedbacks" that provide the potential for expansion. 
These feedbacks, we are told, provide a minimal specification for the nature of living that offers necessary and sufficient conditions to distinguish life from non-living systems.

This paper has similar interests, though it adopts a narrative that is concerned with autopoiesis as a causal process in an "agency" defined as a generic living system. Here, causation enables processes of both internalisation and anticipation, with the latter determining whether the system is living [12]. The term autopoiesis was proposed by Maturana and Varela [13], and while they believed that it could only be applied to living biological systems, their postulated limitation has been overcome [14], enabling living systems to be seen in generic terms. Now, autopoiesis, while a difficult concept [15], is a network of causal processes that enable viable living, and has been deemed responsible for a paradigm shift [16].

If autopoiesis is so important, how can it be conceptualised within the living system architecture? One way is to model the living system as a set of partitioned, autonomous, ontologically related component systems. One might call them the operative system and its metasystem, but where one is concerned with a generalised descriptor in higher-order cybernetics, as in metacybernetics [17], it is more useful to call them anterior operative and posterior strategic-regulatory systems. These are autopoietically connected, with two orientations: the anterior (feedback) trajectory emanating from the anterior system and manifesting strategic and regulative structures $[17,18]$ in the posterior system that are responsible for bounded adaptive strategic movements, and the posterior (feedforth) trajectory emanating from the posterior system, and manifesting operative structures that determine the "look" and functionality of the living system and its operative behaviour. One of the functions of the anterior system is observation. From this, the anterior trajectory cognitively internalises that which has been observed, this resulting in a cognitive model we shall call the ideate (and which provides a basis for the strategic structures that will enable adaptive functionality), residing in the posterior system. Where the ideate influences strategic regulatory structures, the posterior trajectory involves operative anticipation that impacts anterior system structure. Internalisation is a necessary condition that enables the sufficient condition of anticipation to occur as part of living, since the latter is dependent on the former occurring. There are certain attributes of the nature and relationship between internalisation and anticipation within the concept of autopoiesis that require investigation, such as whether and how the posterior and anterior networks individually operate coherently, how the two networks might mutually relate to ensure living system stability, or how one may estimate autopoietic efficacy. To provide such explanations, we shall call on three autonomous but relatable schemas. These are General Collective Intelligence (GCI), Eigenform, and Extreme Physical Information (EPI). GCI offers a pragmatic evolutionary approach that is concerned with a contextually connected purposeful and relatable set of task processes. To explain their relationships, it will be useful to set our narrative within a single framework. Here, cybernetic agency theory [17] will be adopted, where agency is a living system having a population of agents, some of which become members of autonomous subagencies. In GCI, the task processes are assigned to appropriate subagencies that seek a collective "evolutionary fitness." Eigenform is a symbolic approach that is concerned with how observations can be suitably internalised and thus be used as a token to determine future behaviour, and how that which has been internalised can be adopted to anticipate the future. The notion originally comes from Foerster in 1976 [19], adopting the terms eigenvalue related to stability, and eigenbehaviour related to operative structure. However, the story that this tells, while important, is only an entry to explaining living, and to develop it further, the notions of Dubois [20] have been introduced, which contribute to an improved understanding of autopoiesis. Extreme Physical Information (EPI) is an important empirical approach concerned with acquiring information through observation of an unknown parameter through sampling regimes. It will be explained how this can contribute to the idea of autopoiesis, and especially its capacity for efficacy.

In order to be able to connect GCI, Eigenform, and EPI, which are independently derived schemas, it must be shown that they are in some way commensurable. How does 
one do this- that is, what methodological approach is to be pursued? Firstly, it will be qualitative and conceptual, but what tools may be suitable for this? To provide explanation, it is useful to go back to principles of metaphor. Modelling living systems necessarily uses system models, and these are sometimes seen to be metaphors for "reality". Whether they are or not, they enable us to increase our understanding of problem situations and problem-solving processes. Metaphors are important to scientific enquiry [21], and offer us a means by which the use of what is known about direct physical and social experiences can develop an improved understanding of more abstract things (such as work, time, mental activity, and feelings) [22]. Thus, enquiries into living systems occurring through systemic approaches are particularly susceptible to being described as metaphorical because they often occur as a conceptual construction that overlays a real structure. Whether or not they are, they provide us with templates of ideal or representative relationships and modes of being that can be applied to complex activity situations where living systems are deemed to exist. These have certain characteristics that, through the use of cybernetic principles, enable us to explain how and why they function as they do. This gives us a better understanding about the nature of the causal processes that enable them to exist, and a powerful illustration of such a causal process is autopoiesis. Now, a purpose of this paper is to better address living systems so that the modelling process can be improved, enabling the nature of living to be better understood, and autopoiesis is central to this. However, metaphors are double-edged. They can be effective in illuminating new fields of inquiry, but they can also establish apparently motivated links that do not exist at all. The former is an important attribute of the research process, while the latter needs to be addressed by seeking to determine if the motivated links exist beyond ideology.

How does one determine that independent candidate schemas are relatable and commensurable? As long as the schemas are relatable-that is, commensurable with connectable issues-then a configurative approach can be sought. Following Yolles and Fink [23], a configuration has inherent coordinative structures that can respond to the needs of complexity modelling. A plurality of configurations operate as a complex system of interdependencies. As such, they have core orchestrating themes with identifiable characteristics. Configurations can satisfy particular modelling purposes or interests, while simultaneously creating an improved potential to enhance theoretical specificity by modelling detail and generality by relating a plurality of ontologically connected configurations, the result of which can provide elaborated models with an inherent capacity to develop potential. Taking both specificity and generality together improves modelling ability and the capacity to respond to complexity. As a result, a constellation of interconnected conceptual and relational schemas can be embraced through identified explanations, enabling a complex situation to be better understood as a whole [24].

Here, our interest lies in specificity, where a narrative concerning commensurability may be both centrifugal and centripetal. Centrifugal analysis identifies a bounded context with a set of well-known schemas, with an outward perspective that seeks inherent connections that can enrich contextual understanding. As an illustration of this, one may consider a purely cultural context, this involving the schemas of values and norms. An analysis is then able to deliver an emerging narrative describing how the two relate, and therefore contribute to the definition of culture. In the latter centripetal approach, the schemas are not well-known within the frame of reference adopted, and they must first be penetrated to expose specific conceptual commonalities, thereby showing how they relate, this then potentially leading to a centrifugal narrative. An illustration of the centripetal argument can be provided for Foerster's [19] Eigenform, which is concerned with living system stability with respect to observation and the resulting cognitive models, and through exploration, it will be shown how this implicitly connects with autopoiesis. By mutually configuring GCI, Eigenform, and EPI together, an improved understanding will result of the nature of autopoiesis. More generally, it will be shown that each of the three schemas involves processes that are inherently autopoietic, thereby linking them through this characteristic. In the case of GCI, its interest lies in sets of autonomous collective processes in a living 
system that aims at common operative goal outputs that, for Halpin [25], constitute autopoietic processes, and which, when stably converging, provides an indication of the "evolutionary fitness" referred to earlier, and which constitutes autopoietic efficacy. For Eigenform, through Foerster and Dubois, internalisation and anticipation in living systems are either implied (for Foerster) or directly considered (in the case of Dubois), together contributing to the definition of autopoiesis that needs to be efficacious if the living system is to be stable. In the case of EPI, observations create internalised ideates that only arise through efficacious processes of autopoiesis, with parametric measures arising that give propositional support to GCI.

To progress this paper, there is a need to first create a setting for the concept of autopoiesis that will provide a basic appreciation of what is being considered. This setting will be represented in Section 2, where we shall consider some basics of generic living systems expressed in terms of cybernetic agency theory [17]. Here, agencies are taken to be complex living systems that (normally) have a population of autonomous living

Agents in their anterior system, the interactions between them influencing how agency regulates both itself and its agents through its posterior system. There is extensive literature on both agency theory and on the notion of autopoiesis, and we will contribute to the creation of greater coherence in the explanation of how all the characteristics of life (which include such capacities as self-ordering, adaptability to environmental change, selfdevelopment, self-regulation, and a capability for information processing) occur together.

In Section 3, we shall introduce the three schemas and develop centripetal arguments to explain how they are mutually reflective of autopoiesis, the hook that enables them to be related once it is understood that they are relatable. In Section 4 of the paper, the earlier theoretical outcomes will be explored within metacybernetic agency theory, this providing insight into the potential that each has for responding to contexts that may have greater complexity. We shall then relate each of the three schemas, informing a narrative that occurs by setting them in the framework of metacybernetics, this enabling an exploration, in particular, of autopoiesis, efficacy, and self-organisation. A discussion on the developments of this paper will then occur, prior to the conclusions in Section 5.

\section{The Setting}

Here, we shall consider the nature of context as an observable set of characteristics that are represented by subjects in interaction with objects and other subjects, are meaningfully characterised by parameters, and are internalised by living systems enabling them to anticipate the future, so determining behaviour that enables them to maintain their viability. Contexts will first be explored, followed by a discussion about the nature of parameters and their function within a contextual setting; then, the nature of internalisation and anticipation will be considered, which together contributes to an improved explanation of autopoiesis.

\subsection{Contexts}

Agency, as adopted here, has close, if elaborated, association with the notions of social cognitive theory as developed by Bandura [26]. Agency has a population of interactive agents in its anterior (operative) system, and a regulatory structure that is housed in its posterior system (the metasystem), regulation ultimately emerging from agent interactions. The posterior-anterior system have a relationship where they are coupled together through autopoietic processes, de-livering the term autopoietic couple (sometimes also called an operative couple since it couples the metasystem and operative system: Yolles [17]). Agencies are complex entities that can take action or intervention, thereby producing a particular effect. Here, effect relates to an object that, for Kauffman [27], is a symbolic entity that participates in a network of interactions while simultaneously taking on an apparent solidity and stability from these interactions. Now, the nature of an interaction is that it is a mutual or reciprocal influence from which an effect results, so the word effect can be used as a broad term to include objects. However, effects, and therefore objects, are part of a 
context. While, earlier, a context was generally defined as any information that can be used to characterise the situation of an entity, more specifically, it represents the interrelated conditions in which something exists or occurs, and this includes not only objects, but subjects, their interactions, and the resulting effects. Therefore, using the term context provides a short-hand way of describing complex subject-object interactive dynamics and the effects that arise. To describe context, there is a need for parameters, these being crucial in making meaning [28].

\subsection{Parameters}

By parameter, we refer to a set of properties that represent the characteristics of an active context, and the parametric representation of that context will not change unless the context itself does. A variety of parameters may be used to describe and summarise the context, as well as indicating the dimensionality of issues that may require resolution. A parameter is essentially an arbitrary constant that may take a value representative of a contextual characteristic, and it may be qualitative or quantitative. Parameters are used in models, and these may involve finding a set of candidate parameter assignments that together create a scenario, where the assignments can then be tested against prescribed criteria [29], perhaps, for example, using simulation involving iterative convergence [30,31]. Thus, for instance, Beaufils et al. [32] posit a model for cooperative processes in living systems involving iterations of processes that may be able to converge, this leading to cooperative agreements. One class of scenario, called the model scenario [33,34], uses a system model, where variations can be introduced into its unknown or uncertain parameters, this resulting in scenario trajectories that need to be evaluated to determine if they satisfy problem specifications and can contribute to problem resolutions. A qualitative approach may involve a quantitative mathematical model, where the testing criteria may be a parameter algorithm from which parametric values can be determined. Since a set of candidate parameters creates a scenario, the model scenario (using mathematical programming) is able to represent a situation through its set of parameters describing possible and estimable future conditions [35]. Propositions of contextual changes will be represented by parametric changes that deliver new scenarios.

Qualitative parameters may have their own quantitative parameters that describe them. To illustrate this, consider (as did Spathopoulou and Papakonstantinidis [36]) the role of culture in a learning environment, important when it is realised that culture is valuebased and is sensitive to modes of communication that are central to learning. Parrish and Linder-VanBerschot [37] (citing [38-42]), who are interested in the cultural dimensions of learning, provide examples of qualitative learning parameters, these expressed in a value system involving three categories within which there are dichotomous paired values. The categories, and their paired values, are:

(1) Social relationships. These have three paired value parameters: (a) equality versus authority, where determination is sought about how inequality is handled in a given situation, how status is demonstrated and how respect is given, and what interactions are appropriate for those of unequal status; (b) individualism versus collectivism, where it is sought to determine which prevails, the interests of the individual or the interest of the group, and to what degree are interpersonal relationships valued; and (c) nurture versus challenge, where, by equality/authority, it is sought to determine which is the more important set of goals, cooperation and security or recognition and advancement, which achieves better outcomes, and which are supportive or challenging acts.

(2) Epistemological. There are three paired value parameters: (a) stability seeking versus uncertainty acceptance, where determination is sought for how uncertainty is dealt with, being either avoided or accepted, whether structure is seen to be more important than flexibility, and what the status of knowledge is and how it is used in development; (b) logical argumentation versus reasonableness, where one seeks to determine how arguments are developed, which is more important-logical consistency or practical outcomes-and how disagreement is managed; and (c) causality versus complexity, 
where determination is sought about how causality is assigned typically, whether it is assigned to a single, most likely source, or whether it is assigned to the broader context [39].

(3) Temporal. This has two paired value parameters: (a) clock time versus event time, where determination is sought on whether people conform to an external measure of time, or rather permitting the event at hand to unfold on its own time, and whether deadlines or relationships are more important; and (b) linear versus cyclic time, where determination is sought about whether people see time as a path and whether goals are necessary destinations, or if time is seen as a pattern of interlocking cycles into which they step in and out over the course of a life.

It can be argued that the paired values in each parameter are epistemically independent [23], so that any of the above eight parameters can take values anywhere between their dichotomous extrema. Thus, for example, in the case of individualism-collectivism, the parameters may take a value that is either individualism, or collectivism, or indeed some balance between both [43]. Following on from Spathopoulou and Papakonstantinidis [36], culturally blind teaching can challenge cultural value-based worldviews, and may create conflicts that confound teaching aims for a population of culturally differentiable students. Qualitative parameters can be expressed quantitatively, using, for instance, landmark theory [44], where parameters may be differentially scaled into partitions, so that, in the case of the individualism-collectivism parameter, three or more classifications may be identified that have sliding numerical values within predetermined bounds. Thus, consider that individualism-collectivism is scaled to $(0,1)$, with extreme individualism given a value of 0 and extreme collectivism a value of 1 ; then, assuming a linear relationship between the extrema, a perfect balance between them may be 0.5 , though balances may occur that are quite different from 0.5. Over the whole population of students, there will be a variance around the mean for this parameter, which will describe this aspect of the population. A small variance would indicate that cultural differences are likely not very significant. In planning the teaching approach, there will be a need to consider which qualitative parameters are important, given prior knowledge from the quantitative parameters of each qualitative parameter.

Having introduced quantitative parameters, we note that, where they are statistical, the mean is a central tendency of either a probability distribution or of a random variable that is characterised by this distribution, and it smooths out hidden details in any dataset. Variance indicates the variability of a context, and it is a conditioning factor on the mean since it measures the degree of spread of a set of numbers from their mean value. There is another empirical attribute called bias, which refers to the difference between the average predictability from a distribution and a model that is insufficiently parameter-rich, and may thus be considered as an oversimplification that can lead to high levels of error.

Another aspect that may result in bias is where, in complex situations, there are hidden parameters [45], and since context sampling is dependent on the propositions that determine the parametric composition of a context, so sampling of that context may not uncover them (especially if contextual characteristics are subsumed within attributions of noise). As an example in language, Glanzberg [46] explains that hidden parameters imply hidden structures impacting on meaning, and acting as either logical variables or overt expressions of personal taste, as noted by the latter having emotional value [47]. If, in any particular context, there exist hidden parameters, then there will be unobservable elements with unknown relationships that we may encounter. Any approach that seeks to explore such contexts must therefore be sensitive to the needs of uncertainty (as does EPI). When a context is observed, and it is relevant to living, it is internalised through autopoiesis, manifesting it into agency as an ideate, which in turn enables anticipation of the future. Foerster [48] explains the importance of observation to this process that directly connects to cognitive operations, this implying processes of internalisation. However, beyond this, for the ideate to become a token that determines future behaviour (as noted by Foerster), anticipation is required. 


\subsection{Internalisation and Anticipation}

Internalisation and anticipation are causal effects, the functions of which arise from the reverse trajectories of causal-agents, one arising from an anterior system and the other from a posterior system. We can propose that causal effects have functions that can be described in terms of cognitive and context maps. Whether these maps actually exist is not really relevant because their introduction simply provides an explanatory utility that might be represented in other ways. Having said this, the propositions that such maps exist is not new. Internalisation delivers a cognitive map $[49,50]$ from which an ideate is created through assimilation. Such a map is used to store, recall, and decode information about the parametric characteristics of context [51]. Just as language is inherently vague [52], one can deduce that the cognitive maps that underpin language use are inherently fuzzy (lacking in clarity or definition). This reflects on the ideate in its representation of context, and hence implies ideate uncertainty, this deriving from posterior autopoietic processes able to refer to an operative system context map [53-55] that can relate structure with contextual parameters.

Returning to internalisation, it is a causal effect arising from the anterior autopoietic causal-agent, which is able to deliver regulation to the posterior system. In contrast, anticipation is a causal effect arising from posterior autopoiesis, which is able to deliver structure to the anterior system. Internalisation may begin with a context map, and is the result of a recursive (repetitive self-calling) autopoietic process of observing an active context, and, through a cognitive map, creating an ideate that can deliver regulation or regulatory adjustment. Anticipation is the result of an incursive (penetrative) autopoietic process that begins with observing an ideate, and, through a set of processes, delivers a context map from which operative structures derive or are adjusted.

Anticipation is about determining future behaviour [12]. This requires an autopoietically constructed internal model, and it is this that differentiates living systems from non-living ones. Agency achieves an internal model through internalisation of an active context, a process that we have already noted has two states, assimilation and accommodation, and which can be respectively enabled through what Dubois [20] calls weak and strong anticipation. Taking that a cognitive structure is defined by a cognitive schema, then assimilation occurs when an active context is manifested as an ideate to inform this pre-existing cognitive schema. Accommodation changes the cognitive structure by integrating the ideate into the schema and hence into the structure. These two states are important when it comes to determining whether agency is consciously aware or not, and this determines the nature of its cognition. There are two forms of cognition: explicit and implicit [56-59]. Explicit cognition is associated with conscious awareness and is accompanied by implicit cognition. The distinction between the two lies in their processing mechanisms, with implicit cognition being an automatic process and having much less information and operating more rapidly than the rational processes associated with explicit cognition [60].

To illustrate the evolutionary development and distinction between implicit and explicit cognition, it is useful to highlight the case of adaptive artificial intelligence systems [61]. These systems embrace a need for: robustness - the ability to achieve high algorithmic accuracy; efficiency - the ability to achieve low use of resources in computation, memory, and power; and agility - which includes an ability for recognition, and which responds to a need to alter operative conditions based on current requirements. To enhance these attributes, conscious self-awareness is being introduced into processing, storing, retrieving information about the self, and a capacity for individuating - the ability for an entity to distinguish itself from others [62]. Such a development would enable robots to understand their environment and be cognizant about what they do and about the purpose of their actions, making timely initiatives beyond goals set by others, and to learn from their own experiences, knowing what they have learned and how [62,63]. Agencies that are capable of using only assimilation in their internal processes have implicit cognition and a primitive conscious awareness, while those also capable of accommodation 
have explicit cognition and a more developed conscious awareness. Implicit cognition is a general agency property through which information can be processed [64], and it delivers a primitive consciousness connected with agency learning and an ability to modify agency configurations and states in autonomous adaptation [65]. Developed consciousness provides a form of evolution based on stable dynamic support, yielding to strongly anticipative behaviour such as motricity, and enhanced memory and adaptive features, and developed regulation. Setting the idea of primitive cognition into a broader canvas, it has already been explained that Bitbol and Luisi [6] propose that all living systems have degrees of consciousness that enable them to respond to environmental influences. We recall that the most elementary living system has a preconscious condition, and the nature of this is that it has a limited form of assimilation that enables deformation by the irruption of new contextual elements, these being passively incorporated. The next stage is a primitive consciousness that is less passive, involving assimilation that enables agency to evolve and self-regulate, and permits the maintenance of a cognitive status that elaborates the primitive identity of an organised unit. This allows dynamic contextual interaction that is the basic condition for life. Primitive consciousness can be complexified when it becomes involved with dynamics, as occurs in complex organic contexts. An illustration of implicit cognition occurs in the adaptive chemistry of biological systems, where proteins (which participate in system regulation) are able to adapt [66]. Yolles [7] lists six different stages in the evolution of consciousness that starts with preconsciousness, and shows how consciousness evolves from a primitive state to a more developed one as it complexifies. A core distinction between these stages lies in the nature of, and relationship between, assimilation and accommodation during contextual internalisation.

Now, agency has a population of agents which are its subjects. Within a given context, a subject interacts with an object in the environment, and a relationship is observed as an entry to autopoiesis, where observations are acts of identifying, recognising, and noting attributes of reality. For Wildon Carr [67], recognition has a consciousness dependency, and for Thibierge and Morin [68], recognition refers to everything that presents itself to us as reality. Thus, recognition occurs when a context is cognitively internalised, firstly by assimilating it (taking it in); secondly, by complexifying the recognition process (where possible) when that which has been assimilated is now accommodated into the posterior system (metasystem) structure.

That the word recognition has cognition as its route is not accidental, recognition necessarily being dependent on cognition, and since there are two forms of cognition (explicit and implicit), there should also be two forms of relatable recognition. According to Martens et al. [69], implicit recognition involves a context being perceived repeatedly, enabling simple feature detection that gives rise to the automatic perceptual analysis of sensory input from an anterior (operative) system, while explicit recognition involves the conscious discrimination of characteristics associated with rapid perceptual learning in identifying contexts. Both forms of recognition are at work in agencies with developed conscious awareness, but implicit recognition is confined to implicit consciousness in agencies with only primitive awareness. A case of instance is related to supramolecular adaptive chemistry (as an information science) in which molecular information is used by agency in gaining progressive control over its structural and dynamic features during complexification through self-organisation and the drive to life, and where the implicit recognition of molecular events is based on well-defined interaction patterns [70].

\section{The Schemas}

\subsection{General Collective Intelligence (GCI) and Autopoiesis}

GCI can be taken as a schema that seeks to create autopoietic efficacy by satisfying agency needs of welfare and wellbeing (fitness) sustainably. Autopoiesis as a network of processes is decomposed into a pragmatic set of contextually related functional task processes, each undertaken by a team of agents (which we call subagencies). GCI adopts a number of propositions that determine how subagency functionalities can generate collec- 
tive fitness through mutual cooperation. Thus, consider that agency, with a population of agents, some of whom collect together as autonomous subagencies, has functionalities that deliver a task to manifest aspects of an ideate to a contextual situation through agency behaviour. Thus, for example, goals that emanate from the ideate are regulatory phenomena that create autopoietic trajectorial imperatives that exclude agents from seeking alternative imperatives. Thus, a particular goal (which may have been refined through a "higher-order" causal-agent [17]) is extracted from the ideate (by the autopoietic causal-agent) that agency is tasked to manifest materially in a way that complements other related tasks belonging to other subagencies. Interest lies in seeing the subagency assembly as a whole structure that satisfies what the agency needs to sustain. To do this, the subagencies need to cooperate, and this requires collective intelligence. In other words, the subagencies participate in a network of autopoietic processes that need to be coherent together. To determine this coherence, a measure is required called General Collective Intelligence (GCI), and, as we shall see, this comes out to be nothing other than a measure of autopoietic efficacy.

GCI is concerned with group performance, which, for Krafft [71], has a number of attributes associated with it. As illustration, it can be used to increase health and wellness care outcomes through using the individualisation of the relevant care services to increase their capacities [72]. Group performance occurs across tasks and tends to be correlated, so that GCI is a measure of this. A high measure of GCI occurs when a group performs well across a wide variety of tasks, while a low GCI occurs when a group performs badly across a wide variety of tasks. GCI also arises from groups having commitment to cognitive alignment across group goals, accurate shared beliefs, and coordinated actions. GCI adopts the term generic mechanisms, these acting upon living structures that are capable of giving rise to morphogenetic rearrangements. Such mechanisms are nothing other than causal-agents (as with autopoiesis) within which subagencies interact such that there emerge conditions, the effect of which is cognitive alignment-this resulting in performance coherence and collective correlation. If one assigns to a causal-agent the property of efficacy, an efficacious autopoietic trajectory will explain differences in observed correlations in group performance across tasks. Since GCI performance is correlated, this correlation can be measured as group performance for classes of tasks with particular combinations of cognitive alignment requirements.

For Williams [73], GCI enables intelligent agents to become organised into a collective intelligence with improved problem-solving ability. GCI functionality has the potential to increase the impact on collective challenges through decentralised collective efficacious coordination, as opposed to optimisation. GCI is also able to address categories of wicked (or self-perpetuating) problems associated with some group outcomes. Problem-solving processes tend to have an element of centralisation, where attributes of the processes are not inherently decentralised, and where centralised problem-solving processes are not necessarily dynamically stable while serving the group. The most stable dynamic does not necessarily serve centralised interests within the group.

An agency is an adaptive (living system) collective of agents that can act or intervene in relation to an issue, thereby producing a particular effect with, and through, agents acting as autonomous subjects. For Weinbaum and Veitas [74], intelligence is an agency competence defined in relation to an a priori problem domain that involves a formative process of coordinated agent self-organisation. Generic mechanisms are required within agency to enable cognitive alignment across goals, beliefs, and coordinated actions, and, as already indicated, are active through causal-agents. Collective coherence delivers high levels of GCI when agency performances are correlated, with lower levels of GCI when agency performances are less well correlated. Collective coherence is also a function of the network of relationships that agents in an agency have together and with the external world [25]. This is explained through the Extended Mind Hypothesis [75] when two or more individuals can share the same cognitive state, this explaining the emergence of collective intelligence [25]. This cognitive state is delivered through an ideate-internal to the agency and reflecting the dynamic interaction between agency as an observer and its agents (the 
subjects) in interaction with an active context in its environment. This enables agency to anticipate future interactions. The cognitive state also provides a condition that, for Halpin [25], explains the concept of autopoiesis, which supports our earlier proposition that generic mechanisms are causal-agents with an efficacy measure.

Cognition can also be collective when it regulates agency by enabling its agents to become coordinated. Agency regulatory processes function through autopoiesis, as a generic mechanism. Recall that autopoiesis is a network of processes that enables an agency to self-produce cognitively or materially. Under complexity, agency self-production needs to enable intervention strategies that requisitely respond to environmental variety. Collective coherence enables collective ideates to be manifested efficaciously through autopoiesis, i.e., autopoiesis is a causal-agent enabling the activation of self-organisation and an improving capacity for both operative structure and its regulation, leading to requisite adaptation. Autopoiesis operates effectively through collective intelligence, which, on its own, is instrumental by having a non-learning regulatory structure that creates an imperative for behaviour. A measure of agency efficacy should be reflected in any measure of GCI, i.e., the two should correlate. It is because of this that there is necessarily a close connection between a measure of GCI and efficacy. Autopoiesis is an instrumental network of processes that requires autogenesis to help it self-create and thus learn. Different from Bandura's [26] constructivist approach, Yolles and Fink's [23] critical realism sees collective efficacy as the shared capability to organise and execute the courses of action required to manage current or prospective situations.

In learning living systems, there are two types of collective efficacy. Autopoietic efficacy is an attribute of the first-order causal-agent (autopoiesis) that acts as an operative intelligence [76], as originally proposed by Piaget [77], and constitutes an evolving network of process producing autonomous patterns of post-contestation coordinated thought between those agents involved. Autogenetic efficacy is an attribute of the second-order causal-agent (autogenesis) that, again, after Piaget [76], acts as a figurative intelligence involving an evolving higher order of regulation. The posterior system, consistent with personality psychology, may be represented as a "generic personality," defined as an assembly of (implicit or explicit) cognitive characteristics that are subject to cognitive biases and pathologies that may strategise agency adaptation while simultaneously engaging in regulation. Cognitive biases can be thought of as parameter-poor cognitive processes that arise through unbalanced traits [23], similar to the situation described earlier when discussing the Parrish and Linder-VanBerschot [37] notions of individualism and collectivism.

Plural agencies, those composed of a subagency collective, operate through the regulatory control of a post-posterior system (i.e., one that is superior to the posterior system, resembling a cultural system that influences a personality system). Where agency is a plural entity, personality is normative [76], the norms being regulatory elements that emerge from agent interactions. Analogous to the distinction between tight and loose cultures [78], the strength of regulatory control [79] may be usefully described to be tight or loose. In distributed agencies, loose post-posterior systems provide weak regulatory control, while in centralised agencies, tight post-posterior systems provide strong regulatory control. GCI is deemed to operate with distributed subagencies experiencing weak regulatory controls, when subagency coordination may be a function of negotiation. As an example, a distributed organisation tends to operate with a loose culture having weak norms, an emotionally high tolerance to deviant behaviour, few rules or standards, and beliefs which are relatively heterogeneous [80]. The use of the term emotion does not necessarily imply explicit cognition. In agents limited to implicit cognition, implicit emotion may be deemed to be any process that operates without explicit intentions, and modifies the quality, intensity, or duration of immediate responses to internal perturbations that may derive from malevolent external influences.

In agencies with efficacious self-production, the measure of GCI will be high. In this case, autopoiesis will need to satisfy at least one condition: that any inherent fuzziness of the selected characteristics of a context with parametric representation must be minimally 
bounded by some error function. How such fuzziness may arise is due to the means by which information is acquired, no matter how advanced the techniques for analysis may be. This can be seen from the work on GCI undertaken by Krafft [71].

To determine a value for GCI, modern multi-agency approaches can be adopted in which parameters and constraints take up a central position. Consider that an agency has an autopoietic network of processes, where each network element is a manageable logical entity in a distributed structure. Each network element belongs to an autonomous adaptive subagency (with its own population of agents), where the network element is seen as a property of a subagency that is associated with a particular functionality. The multifunctional nature of autopoiesis therefore implies, through the network of processes that defines it, a complex interaction between subagencies. The relationship between subagencies can now be examined from a complex information processing perspective, and computational theory [81] can be applied through the use of algorithms. For this, information processing systems can be solved as particular computational tasks through the particular implementation of specific algorithms. Krafft [71] explains that, to do this, there is first a need to identify three levels of complexity for a given context: computational, algorithmic, and implementation. The computational level seeks to question the teleology/purpose of a functionality, and the algorithmic and implementation levels seeks to respond to this initially by formulating and then applying algorithms. The way in which the interactions are solved is by analysing the abstract phenomenon of GCI as if it is a computational property of varied subagencies across an agency landscape. This involves the exploration of cognitive parameters such as beliefs, goals, and actions under strict alignment conditions.

Underlying the GCI approach is that the autopoietic network of processes is defined in terms of a plurality of autonomous adaptive subagencies that populate the agency, each delivering its functional process. The overall approach conforms to the interactive structure of a sustainable plural ecosystem as described by Yolles $[7,82]$ that is not a single adaptive system, but rather the assembly that seeks to develop "as an evolutionary landscape that embraces natural selection, this moderated by an understanding of agency self-organisation" ([82] (p. 12)) that seeks both viability and sustainability. This requires that the dynamic in an agency ecosystem composed of subagencies can be anticipated, thereby facilitating an appreciation towards the needs of sustainability. For Vickers [64], appreciation is important to the value-based perspective that is consistent with the networking needs of benefit, and for sustainability. This requires that subagencies need to be prepared to respond to interactions through a valuing of processes that are self-reinforcing, and that involve an examination of their dynamic trajectories and subordinate amenities that enable them to satisfy their functionalities [83]. Examining the viability of development in an evolutionary landscape can thus be seen as a process of natural selection, moderated by an understanding of subagency self-organisation. This, for instance, embraces decision structures and processes that need to respond to appreciative inquiry [84].

The evolutionary approach towards sustainability and viability relates to agency wellbeing (emphasising cognitive and affective/emotional health) and wellness (focused on physical health), the two together indicating fitness (the ability to cope with the environment). As Alexiou [85] explains, fitness has arisen through an evolutionary approach to games, providing an alternative to utility maximisation/optimisation. There is a limitation to this approach in that the efficiency of the competitive strategies that exemplifies an evolutionary multi-agent design is a stable coordination strategy that creates a learning opportunity essential in achieving cooperation in conflicting situations.

This must recognise that agency viability within GCI embraces the maximisation of subagency fitness [86]. This is a required condition common to evolutionary processes. Williams $[73,87]$ considers some propositions that can satisfy subagency fitness maximisation for GCI. Since each subagency has a functionality, collectively, they must interact according to certain principles of decentralised cooperation so that they are able to maximise fitness outcomes for each. Thus, functions must be prioritised according to principles 
that enable this. Now, an autopoietic network of processes is defined in terms of active subagencies that need to satisfy certain adaptive principles. These include a condition of centralisation, and three classes of decentralisation, which include process operations, relative value determination, and definition of fitness, based on Williams [73]:

- Centralisation:

- Process selection

selected processes to be executed to satisfy fitness in achieving collective outcomes so that it is possible to maximise those outcomes.

- Decentralisation:

- $\quad$ Process operation

is purely Peer-to-Peer, with no third-party involvement;

$\bigcirc \quad$ is decentralised, where process activities have role assignations;

is user-centric, with no third-party information;

$\bigcirc \quad$ is massively collaborative, with no predefined limits that scale participation.

- Relative value determination

$\bigcirc \quad$ of work, universally to enable open participation by subagencies in collective processes;

of resources, enabling more open sharing of those resources, thus enabling collective processes.

- Metric of fitness definition for

subagency participation, through openness, enabling subagency substitution for others able to better enhance viability in the cooperative process; subagency process, enabling selection of the best process in which to participate.

Subagencies involved in autopoietic processes may participate in collective cognition, or operate as functional components of cognition. In both cases, there is a centralisation constraint to ensure adherence to the principle of cooperation. As already indicated, this sets up GCI conditions for a multi-agent modelling approach that can enable scenario creation and the use of simulation techniques [85]. However, in the end, factors such as hidden parameters and dynamics (especially with respect to personality pathologies, idiosyncrasies, and cognitive biases) may occur. Instances might be declared subagency beliefs, goals, and actions having unexpressed caveats that perturb parameter representation and corrupt problem definition.

\subsection{Eigenform, Internalisation, and Anticipation}

Eigenform began as a cybernetic theory of observation that, using symbolic logic, describes how an active context is observed and how this results in eigenvalues and eigenbehaviour. This demands the explanation within the framework being used here that it occurs through internalisation resulting in an ideate, the internalisation occurring through autopoiesis. Autopoietic efficacy is an indicator of how capable autopoietic processes are in internalising context. When context is sufficiently well represented, autopoietic stability results together with eigenvalues, enabling agency to stably self-produce components of itself as eigenbehaviour, an operative structural dimension of agency which then provides the potential for behaviour. With stability, the ideate can now be used as a token to anticipate future agency behaviour. Posterior autopoiesis is responsible for recognising the ideate and delivering anticipation to agency. The background to this comes from Piaget's research into the cognitive development of children.

Piaget [88] was interested in better knowing the abstract learning process by exploring the connection between an observer and the contextual subject-object relationship. He illustrates the process involved in such learning with a child counting pebbles. To count these, the child puts them in a row and progresses a count from 1 to 10 . On finishing the count, he repeats the process from another direction, similarly to find 10 . This discovery enthuses him to reconsider what has happened, and he puts them in a different arrangement 
and continues counting, always to find 10. Thus, he discovers a property of actions, not of pebbles. The interaction between the boy as subject and the set of pebbles as object defines the context, where only the relationship between the pebbles and subject changes. When the context is internalised, the subject no longer needs to count pebbles, but rather combines the operations symbolically, thereby creating a logical process in which the actions are collectively coordinated. This in turn results in an abstract structure that may be applied to contexts other than the counting of pebbles by the subject.

Piaget's construct was further explored by Foerster $[19,89]$, who noted that when an observation is internalised at time $t$, it becomes a token of the past at time $t+1$. He elaborated on this using recursive symbolic logic to describe the process of observation. That is, observation explicitly recognises and notes characteristics of context, this requiring awareness as a consequence of explicit cognition. It involves agency autopoietic couple stability that occurs when the ideate is sufficiently similar to the relevant context to enable stable self-production. An agency with autopoietic couple stability develops an eigenvalue which, through autopoiesis, enables stable behaviour. Eigenbehaviour occurs in agencies that have eigenvalues [89] when the autopoietic couple is stable, this being stable selfproduction. Stable self-production is necessary to enable agency to develop coherent operative structures from which stable behaviour can be enabled. These structures may be virtual, as in the case of organisations. In the case of biological organisms, they can deliver physically observable agency distinctions of forms that can distinguish between species through generic patterns, and individual identity through specific variations in those patterns. In Foerster's terminology, this is expressed in terms of eigenbehaviour that is responsible for operative structures. This might result in physical patterns such as the coats of animals or the shell of snails or mussels [90], and delivers potential for behaviour.

Foerster represented this recursive process of observation using symbolic logic that ([89] (p. 371)) explains results in a symbolic logic in a recursive equation that has an implied solution giving an eigenvalue, and where it is postulated that there exist:

"Solutions for an indefinite recursive equation based on Jean Piaget's recursive structure of implications describing an observer's account of an interaction between a subject and an object. This equation basically asserts that what is observed at one particular time (obs) is the result of a cognitive/sensory-motor operation (COORD) on the previous instance of observation."

Varela [91] extended Spencer Brown's [92] "Calculus of Variations" into a calculus for self-reference through autopoiesis [93], and Kauffman [94-97] similarly applied this to Foerster's Eigenform, delivering self-referential equations and supporting the idea of autopoietic couple stability. Foerster's formalisation of Piaget's learning process enabled this stability to be defined as a precondition for an eigenvalue, with eigenbehaviour a stable consequence. Briefly, Rocha [89] explains that Foerster's approach concerns an observer's account of an interaction between a subject and an object, this principally describing the process of internalisation that occurs through anterior autopoiesis. Rocha further explains Foerster's approach in terms of a symbolic equation that asserts that an observation at one particular time $\left(o b s_{t}\right)$ is the result of a cognitive (or sensory-motor) operation COORD (an autopoietic imperative that, in due course, results in an ideate) on the previous instance of observation $\left(o b s_{t-1}\right)$ :

$$
o b s_{t}=\operatorname{COORD}\left(o b s_{t-1}\right)
$$

This is a recursive expression that has no starting position or initial condition. As such, any particular instance of observation will be the result of an indefinite successive set of cognitive operations. The observables do not refer directly to real world objects, but are instead the result of an infinite cascade of cognitive and sensory-motor operations (subsumed in COORD). There are solutions $\left(O_{i}\right)$ to this equation which do not exist in a strictly mathematical sense, since there are no initial conditions. However, they do represent an autopoietic couple stability in the chain of COORD operations. This refers to those 
values which maintain their structure (or operation, or function) when cognitive operations on them are repeatedly performed as the equation pursues its indefinite recursive chain:

$$
O_{i}=>o b s_{t}=\operatorname{COORD}\left(\operatorname{COORD}\left(\ldots \operatorname{COORD}\left(o b s_{t-n}=O_{i}\right)\right) \ldots\right)
$$

(for $n=1,2,3, \ldots, N$ ). Rocha further explains that for a long succession of cognitive operations, when there is no change in the active context from which $o b s_{t}$ arises, then this indicates that a convergence has arisen to an eigenvalue for which $O_{i}$ is its representation. Such eigenvalues are self-defining/self-referent in their autopoietic couple stability, which, through the operator COORD, implies a complementary relationship (circularity, closure) between eigenvalues and cognitive operators, where one implies/defines the other. As Rocha [89] explains, eigenvalues represent the externally observable manifestations of the (introspectively accessible) cognitive operations (COORD). Eigenbehaviour is thus used to define the consequences of self-production deriving from autonomous cognitive systems, which, through cognitive closure, give rise to perceptual regularities.

By cognitive operations is meant the inclusion of autopoietic processes that are responsible for both internalisation and anticipation. Since $o b s_{t}$ are agency anterior autopoietic functions, then, after Rosen, agency is only living if it can anticipate. In this case, COORD is necessarily not only a function of the past and present, but also of the future, in order to entail anticipation. However, this anticipatory nature of COORD is not considered; while it involves $o b s_{t-n}$ in recursive mode, one might suppose that it should also involve, say, $o b s_{t+\eta}($ for $\eta=1,2,3, \ldots, \eta)$.

Reflecting on this, and noting comments made earlier in relation to internalisation and anticipation, one can consider that autopoiesis is a dual process in that it has: (1) an anterior autopoietic network of processes relevant to the internalisation of an active context relevant to eigenvalue formation and agency regulation, and (2) a posterior autopoietic network of processes relevant to anticipation and eigenbehaviour. Adopting principles elucidated in GCI, each network may itself be considered as a set of interactive subagencies (delivering its functional processes), which mutually interact and operate as an autonomous distributed collective subagency. The collective may be stable in its own right in that it satisfies a functional purpose to manifest inputs into requisite outputs, despite any possible perturbations that need to be overcome (indicating stability) that further differentiate a context from its representative ideate. This general definition of autopoiesis can now be applied to both anterior and posterior trajectories. Thus, anterior autopoiesis is responsible for transforming an observed context, manifesting it into a requisite ideate that can deliver eigenvalues. In contrast, posterior autopoiesis is responsible for observing the ideate, and manifesting this into a potential that delivers eigenbehaviour when stable. In the anterior case, it would therefore appear that Equation (2), when solvable, creates local anterior autopoietic eigenvalues. In the posterior case, an equation is needed that, when solvable, creates posterior autopoietic eigenbehaviour. That eigenvalues can result in eigenbehaviour in Foerster's theory is really presented as an opaque and mystical phenomena due to its lack of concrete description, and we recommend that it should be seen as an operative structural phenomenon in an anterior system that provides potential for behaviour.

The current problem at hand is therefore to find an expression that can represent posterior autopoiesis that, when solved, can indicate stability. A resolution for this comes from Dubois [98], who begins by first reformulating the recursive expression given in Equation (2) as:

$$
y(t+1)=R(\ldots, y(t-2), y(t-1), y(t) ; A)
$$

for $y(t)=o b s_{t}, R$ is the recursive function COORD, and $A$ are a set of parameters that are representative of the context, and where, for any given scenario, $A$ is to be selected. To know the function $R$, the values of the parameters $A$ and the initial conditions..., $y(-2)$, $y(-1), y(0)$ at time $t=0$, and the successive states $y(t+1), y(t+2), y(t+3), \ldots$, must be determined for the time interval $\Delta t=1$. 
Now, moving on to anticipation, Dubois explains that there are two types, weak and strong, where:

- Weak anticipation occurs when the ideate is an informational model of the active context, and this arises from exo-anticipation, that is, agency anticipation about the external active context that creates expectations for eigenbehaviour and hence the potential options for future agency behaviour.

- Strong anticipation arises from behavioural endo-anticipation, where the ideate becomes embedded in the agency structure which can then determine eigenbehaviour and the potential for future agency behaviour.

We may observe that exo-anticipation is an informational subordinate to agency structure associated with assimilation, while endo-anticipation is structural and a consequence of accommodation. To represent anticipation, Dubois introduces the term incursion. This is directly associated with anticipation, and is a fundamental living agency property that enables it to anticipate, and thus embrace, an evolving future.

Dubois now sets out an expression for incursion that satisfies Rosen's idea of anticipation, incidentally implying posterior autopoietic processes. He thus formulates a new discrete (and later a continuous) expression for weak anticipation that depends on prior assimilation, where the current agency state at time $t$ is a function of its past states $t-3$, $t-2, t-1$, its present time, $t$, and its exo-anticipated states at future times $t+1, t+2$, $t+3, \ldots$

$$
y(t+1)=A\left(\ldots, y(t-2), y(t-1), y(t), y^{*}(t+1), y^{*}(t+2), \ldots ; A\right)
$$

where the variable $y^{*}$ at future times $t+1, t+2, \ldots$ is determined through agency exoanticipation, for some $A$. Dubois notes that $y^{*}$ can be an expectation, and there appears to be no reason that this should not have a probabilistic origin, perhaps from theory similar to that of EPI.

Essentially, the same expression is used with a minor adjustment to represent strong anticipation, assuming prior accommodation, where a discrete incursive agency condition (within which the current state at time $t$ ) is a function of its states at past times, $\ldots, t-3$, $t-2, t-1$, present time $t$, and its states at future times $t+1, t+2, t+3, \ldots$.

$$
y(t+1)=A(\ldots, y(t-2), y(t-1), y(t), y(t+1), y(t+2), \ldots ; A)
$$

and where $y$ at times $t+1, t+2, \ldots$ for the future is determined through endo-anticipation, so that agency structure is indicated within the equation. The incursive agency is selfreferential, and able to determine its future states through endo-anticipation as a consequence of accommodation and from itself, rather than from a model-based expectation connected with assimilation. That solutions can be found for Equations (4) and (5) indicates the existence of both eigenvalues and eigenbehaviours.

\subsection{Extreme Physical Information and Autopoietic Rationality}

Extreme Physical Information (EPI) is concerned with gleaning the nature of an observable context from the observation of samples from it, given a set of relational parameters that have quantitative parametric descriptors that broadly describe it. It acquires contextual information used to create an ideate, the acquisition being an autopoietic process with an efficacy measure, this indicating how good the information is from which the ideate is formed. It can thus be used to indicate the goodness of similarity between the ideate and the observed active context, and hence it is an indicator of stability.

EPI is intimately connected with Fisher information theory, which was originally concerned with statistics, though it may also be framed within the context of observation of context and its analysis. Every one observation of a real effect is a single data value (e.g., a time occurrence, a position occurrence, an electron charge occurrence), which is governed by the statistics of the effect-that is, the probability law governing the effect. The effect is fundamentally statistical, and as such can be analysed by statistical theorems. These 
are, in turn, mathematical in nature. Statistical data can only be properly analysed by the use of such statistical theorems. Many of these are due to the renowned statistician R. A. Fisher [99], including his invention of "Fisher information," as it is called nowadays. He was the father of bio-statistics, and the latter of course include those involving humans and their observations. In EPI, Fisher information values will be represented as a matrix of values that characterise a matrix of channels, each of which corresponds to a contextual parameter. However, in the case considered here, we limit ourselves to only one such channel that characterises the contextual "state" (however one describes it, often its mean value) of a one-dimensional system (such as a vector). Thus, here, the Fisher information matrix is manifested as a single scalar number.

Exploring the agency relationship between ideate to context in terms of information, there is a principle that if a maximum amount of possible information can be extracted from a context, then there is likely to be autopoietic couple stability. This is consistent with the idea that perturbing observation through pathology, idiosyncrasy, or cognitive bias can corrupt the acquisition of information that is representative of a context, this bounding what can be acquired.

This is consistent with Roy Frieden's [100,101] EPI mathematical theory built on the principles of Fisher information. It is a computational approach that originates out of the observation of data from an unknown phenomenon. That is, observation includes not only "seeing" or "visualising" the phenomenon but quantifying it. A statistical dynamics approach, it provides a way of explaining how well one can determine a physical law, given a fixed amount of data from it.

Suppose that the data values $y$ each obey $y=A+x$, for some $x$ and with $A$ unknown parameters defining the system's state (e.g., its mean energy level). This is a simpler expression than provided by Equation (3), but there would appear to be equivalence if one considers that EPI does not need to demonstrate stability in terms of repeat observations, and is normally interested in a single observation that eliminates the need for recursive time periods. With the data $y$ known, $A$ is computed via some required function of the data $y$ (for example, as their simple average). The apparent "noise" values $x$ is purely a phenomenological fluctuation that actually define the physical effect driving the system. It obeys some probability distribution $p(x)$ describing uncertainty in knowledge of the effect. The data $y$ also ultimately determine the total amount of information $I$ carried by the system, via the EPI principle $I=$ maximum. This maximisation is carried through algebraically, subject to all known constraints on $p(x)$ (such as normalisation, perhaps the mean value $\langle x\rangle, \ldots$; see below). Knowledge of parameters intuitively depends on how narrow the distribution $p(x)$ is, or, equivalently, how much information is collected in the data.

The data are, as a result, often termed "smart". This is most apt in quantum systems, for which the mean-squared error obeys directly $1 /$ I. On this basis, the higher the information level $I$ is, the smaller the error is in determining the system state-an intuitively correct result.

EPI is a general theory that can operate together with metacybernetics $[17,23]$. EPI, as with Eigenform, is also an approach that uses the idea of observation, but we can differentiate between them by again recognising the distinction between implicit and explicit recognition. Eigenform is an act of implicitly recognising and noting attributes of context, while in EPI, it is an act of explicitly recognising and noting attributes of context acquired through measurement (as above). Inherently, then, the two are commensurable since explicit recognition involves implicit recognition. In both cases, the character of a context is delivered, from which an ideate derives. In EPI, explicit observation measures active context, subject to theoretical prior knowledge of that context. EPI and metacybernetics share certain premises. Metacybernetics is a general theory of higher-order cybernetics with a substructure of cybernetic axioms, while EPI has an axiomatic substructure deriving from Fisher information axioms. 
Stability in the autopoietic couple is all about order. If the context is coherent and therefore is ordered, then there should be a sufficiently close, relatable order in the ideate. Fisher information provides an approach that is capable of determining what it is that constitutes "sufficient" order. Providing that one can identify parameters that can adequately identify a given context, then one can adopt statistical methods to determine the state of disorder of that ideate. Essentially, Fisher information is an indicator of the expected error in a "smart measurement". Consistent with critical realism, EPI is an observer-based theory where the observer is not in any way "ideal," and where there is imperfect observation-that is, observation with added noise/fuzziness. Nevertheless, as will be seen below, EPI may be used to find the physical laws giving rise to faulty observations. This is a case where observations are imperfect, but lend themselves to analysis in being simply regarded as "additive fluctuations" in the values of measurements.

Here, we adopt EPI to explore post-nonclassical autopoietic couple stability. There are some caveats relevant to EPI. The first caveat comes from it being a general mathematical theory that is concerned with statistically repeatable effects. The second caveat is that it assumes known context for the observed effect, at least as well as possible under complexity. Thus, knowledge will be limited to observable relationships between the entities that are seen to compose a context. The third caveat is that it is able to build theories, i.e., mathematical models, and its limitation centres on the measurement of contexts. It has thus been successful in replicating existent mathematical theories from its own substructural axioms that relate to Fisher information in a variety of fields, including quantum mechanics, classical electromagnetic theory, statistical mechanics, and gravitational theory. EPI has also been used to build theory in other disciplinary areas such as ecology and economic and social science [102-104].

Consider that the context can be recognised by the information bound to it, this being represented by $J$, and that $I$ is the information acquired from the context that reflects the information content of the ideate. These numbers $I$ and $J$ are values that come from Fisher information [100]. The relationship between $J$ and $I$ is an effective flow of information given by

$$
J \rightarrow I
$$

Consider now that observation is generally imperfect. Suppose that the context at a specific point in time can be specified by the value of a single parameter $A$ that is indicative of a scenario, where $A$ is generally a fixed and definite vector of numbers. Agency wishing to gain information about the context observes it for its value $A$. This is indicative of the set of relationships between parametric components $A$, which deliver the observed values $y$, resulting in the ideate $\Phi$. The relationship between these was earlier indicated, but it is useful to now set this centre stage. Practically, $y$ is not representative of $A$ since there is also a random value $x$, where

$$
y=A+x .
$$

Now, $x$ is an intrinsic fluctuating effect indicative of an anomaly such as observational error. Consider now that the totality of possible intrinsic fluctuations $x$ define a probability law $p(y \mid A)$. Knowing $p(y \mid A)$ can therefore provide some indication of the quality of contextual observations that deliver the ideate. In the end, EPI is concerned with identifying the maximum attainable change in Fisher information. This is subject to the constraints that are determined from a problem context. If one has confidence in the constraints, then there will be equal confidence in the solution for $p(x)$. Now, the value $I$ for observation $y$ is defined to obey Fisher Information, as

$$
I=<[d / d x(\log (p(\boldsymbol{y} \mid A)))]^{2}>
$$

where $d / d x$ is a mathematical derivative of its log argument with respect to $x$, and the brackets $<>$ indicate an expectation-that is, multiplication by the probability law $p(y \mid A)$, called the "likelihood function" in statistics. It defines the probability of each possible 
observation in the presence of an ideal parameter value $A$. Interest now lies in estimating the probability law since this defines the unknown effect under study.

The expectation $<>$ may be evaluated as a simple integral over all $x$ (Frieden, 1998). Hence, if $p(y \mid A)$ is known, so is $I$, and differently shaped laws $p(y \mid A)$ give rise to different values for $I$. Equation (6) holds for the particular law $p(y \mid A)$ giving rise to the data. If the wrong law is assumed, the answers will be wrong since the wrong prior knowledge is being used. The law holds regardless of any error that might occur in the relative connection between the observation and the context. Such an error occurs if, for example, the observer is being tricked, and the data observed really do not arise out of the scenario under study, or, alternatively, if the prior knowledge to be used does not arise out of that scenario. In the latter case, just different (and correct) prior knowledge must be used. The need is to avoid errors in prior knowledge (which might include mean and variance).

In evaluating Equation (8) for various laws $p(y \mid A)$, it becomes apparent that $I$ is a measure of the width of $p(y \mid A)$. For example, if $p(y \mid A)$ is a normal law, its use in Equation (5) gives $I$ as simply 1 divided by the variance. The variance is roughly the squared width of $p(y \mid A)$. Hence, the wider the law $p(y \mid A)$ is, the smaller is the information value. The wider/broader the probability $p(y \mid A)$ on the fluctuation is, the more "random" the values of $x$ are, and so the less accurate is the estimate for the parameter $A$ from an observation $y$. In this case, there would be an expectation that $I$ would take a small value indicating stability reduction, so that $\Phi$ is not a good representation of context. This is precisely what Equation (8) gives in this situation. As such, Fisher information I measures the information about an unknown parameter in the context representing a typical data value $y$ as an indicator of the information possibilities that might be extracted by observing a context. As such, it is capable of inferring hidden structure.

Consider now that the fluctuation $x$ satisfies $p(x)$. The shape of $p(x)$ is important, and the wider/broader it is, more unpredictable is a value of $x$, and hence of $y$. This gives rise to inadequate observations $y$, which are then autopoietically delivered to the ideate. Since observation is part of the autopoietic process, the autopoietic couple cannot be considered to be stable in terms of its anterior trajectory since the context is not well represented in the ideate. In this case, we can say that the autopoietic couple is not well ordered, the anterior autopoiesis having poor efficacy. From Equation (7), the information I allows the degree of autopoietic efficacy to be quantified, i.e., represented by a number. This is normally expressed as a measure of entropic disorder $H$. Now, $H$ relates to $I$ if the probability law $p(x)$ is such that the level of disorder monotonically increases with time (called the Fokker-Planck differential equation: [76]). The level of disorder is determined by the level of entropy $H$, which must increase (by a relation $d H / d t \geq 0$ called the "Boltzmann H-theorem" or the Second Law of Thermodynamics.). Correspondingly, the Fisher information decreases as:

$$
d I / d t \leq 0
$$

This is a "Fisher $I$-theorem" that corresponds to the "Boltzmann $H$ theorem" $d H / d t \geq 0$. As a consequence, both measures I and $H$ change monotonically with the level of disorder. Hence, the Second Law is described by Fisher $I$ as well as by entropy $H$. This means that the use of EPI often precludes necessary use of the concept $H$.

As a result, it may be said that a necessary condition for there to be order in the autopoietic couple is that the value of $I$ is large. In quantitative terms, this can be stated as follows: the mean-squared error $\mathrm{e}^{2}$ in estimating the state of the observed system from its data goes as $1 / I$. This is called the Cramer-Rao inequality. A small value of $\mathrm{e}^{2}$ indicates a low level of disorder. Thus, in summary, if the context is in itself a complex coherency, and if it is well represented by the ideate, then the ideate must also be coherent in itself with a large value $I$, and the ideate is well ordered as a reflection of the characteristics represented in the relationships of the context. This is the same that occurs in autopoietic couple stability in Eigenform theory, and so the two are equivalent. 
Now, in EPI, the efficiency with which $I$ is autopoietically determined from $J$ is given by the expression

$$
I=\kappa J,
$$

where $0 \leq \kappa \leq 1$, and $\kappa$ is a constant that measures how well information is acquired from the context and delivered to the ideate. In EPI, $\kappa$ is a scenario-determined qualitative parameter indicative of the minimum loss in representing the $J$ bound information as acquired $I$ information. However, since this efficiency is a reflection of the capability of agency to autopoietically represent the context as an ideate, $\kappa$ may also be seen as an autopoietic efficacy measure, the value of which is also indicative of the agency capability potential to achieve autopoietic stability. If one considers a classical physics scenario, then the value of $\kappa=0.5$, while in a quantum physics scenario $\kappa=1$, meaning that $I=J$ (no information is lost). No matter the scenario, the value of $I$ will still have a range able to indicate autopoietic efficacy. This condition also suggests that the ideate is entangled with the context so that no information is lost, and for any scenario, all the contextual information is now characterised in the ideate. The result is unequivocable stability. In complex situations, there are hidden characteristics and relationships, not all of which will be inferred, so $\kappa=1$ is unlikely. However, in a learning situation, the value of $I$ can approach that of $J$ [105]. To explain this, consider a context with hidden attributes from which wicked problems emerge. If inquiry methods are applied to the context to enable an observer to learn about these hidden attributes to better enable problem resolution, and a new observation is made, then, for a given J, the value of I can increase. However, in wicked problems, new characteristics and relationships will arise after an intervention, thus complexifying the context, so that $J$ changes and no advancement has occurred.

There is another issue that needs to be considered, for which Gatenby and Frieden [106] provide relevance. They discussed the problem of carcinogenic growth in biological systems, where cancer does not grow out of one source but out of many. That is, many nearby cancerous cells flood a given functioning (healthy) cell with their lactic acid, starting its own cancer growth, and this is the way in which it spreads. If there were only one source, then, at most, $I \leq J$. However, for two sources, at most $I \leq 2 J$, and for $n, I \leq n J$, giving $\kappa \leq n$. Now, in cancer spread, physical effects allow for selfishly beneficial growth, with cancer growth being enhanced by outside systems to the detriment of the total system in space-time, since the system dies. This enables one to reflect on social situations in which there is a group of $n$ autonomous subagencies interacting together cooperatively, all making information contributions. This is a situation that is obverse but reflective of cancer growth, again giving $\kappa \leq n$. This, however, also says something deeper, that there is a very basic difference between physical effects (where $\kappa=1 / 2$ in classical situations, or $\kappa=1$ in quantum situations) and social effects in which, in an agency, agent ego dominance is replaced by collective sustainability through cooperation. Such social effects allow for mutually beneficial alliances $(\kappa>1)$, where agency growth is enhanced by other external agencies, both of whom benefit from longer life in space-time. This is yet another demonstration (originally explored by John Forbes Nash Jr.) that cooperative processes can trump egocentric tendencies [107].

To explore this further, suppose now that, in the autopoietic process, the $n$ autonomous self-organising subagencies each have a certain processing purpose that they are pursuing, and to assist collective cooperation, they maintain a lifeworld interaction (lifeworld being the collective intersubjective pool of perceiving through which meaningful thematic communication occurs [108]). In other words, we are looking at effects that are the result of $n$ subagency interactions. In terms of EPI, since the subagencies are functionally autonomous, their individual information values maximally add. This means that any collective policy arising out of their functions would convey maximum information about subagency policies and the parameters contained within. If the subagencies report their decisions to a central agency, then the overall authority makes a summary assessment for fitness-related outcomes that incorporate the proposals of the $n$ subagencies, with feedback recommendations. This situation is related to that of cancer growth, when one cell invades 
another with lactic acid, but instead of acid, there are language-based conversations, noting the realisation by Glanzberg [46] referred to earlier, that language may have hidden parameters that impact on meaning. The intention over the whole agency for the assembly of conversations is that subagencies are able to reach agreement on their actions such that their fitness (physical wellbeing and mental wellness) is maximised. This supposes that the central agency to which these subagencies report reaches an "agreement" based on these reports. The total information collected by the central agency from the subagencies is thereby maximised. Here, then, the context is not so much subject-object interactions, but rather subagency-subagency interactions. Following from Glanzberg, and recalling that two types of parameter are logical variables or overt expressions of personal taste, it appears likely that both are in some way identifiable and measurable, the latter at least by content analysis and related statistical analysis across an array of subagency linguistic constructions [109]. Hence, policy as a linguistic construct must have within it statistically measurable parameters, some of which may be hidden (and possibly inferred by EPI). Thus, following this argument, policy parameters that are reported in the conversations can be evaluated probabilistically. They might then be associated with such attributes as goals, beliefs, and coordinated actions, where, for instance, goals might have parameters relating to who, what, where, and when. Let us speculate on the approach that might be adopted, which is very broadly related to the analysis of personality by Yolles and Fink [23]. A set of parameters for goals would be identified that are relevant to the whole assembly of subagency functions. These could be set up as a cognitive map, together with their correlations. A statistical analysis might then be applied to the assembly of communications relevant to goals. Applying a statistical correlation approach, joint probability distributions emerge, and these might be suitable for an EPI analysis.

In a simple qualitative illustration, consider that the subagencies represent election voters in various sections of a country. Each such subagency counts votes independently of the others. Next, each subagency reports its vote count to a central government agency with overall responsibility for the election process. In usual democratic voting situations, each subagency vote tally is accurate and is independently sent to the central government agency, where it is assimilated (thereby obeying maximum Fisher information). In this case, the total vote count by the central government agency contains maximum Fisher information about votes in all sections of the country. In this manner, democracy is a systematic program that represents the maximum information voting positions (indicative of opinions) of the overall population of a country.

The efficacy effect of Equation (10) derives from a premise from Immanuel Kant. This is that the acquirable level of information $I$ about a context present in data of the total information level $J$ obeys $I \leq J$. Depending on the effect, either some or all of its information can be obtained. Expressed as information change,

$$
K=I-J \text {. }
$$

can indicate an information loss if it is negative. However, this does not necessarily mean that the autopoietic couple is unstable. For example, conventional simple systems of classical mechanics, which have long served mankind as paradigms of stability, obey $I=(1 / 2) J$ so that $K=-(1 / 2) J$, a loss. By Equation (11), $K$ is always zero or negative, indicating that it is generally an information loss, so that the set of parameters characterising relationships of a context in $\Phi$, if correct, are insufficient.

When anterior autopoiesis is perturbed in some way, it can cause the observation of the bound information $J$ to be perturbed, thus perturbing $I$, and we have instability unless the autopoietic perturbations can be overcome. With stability, there is no loss of information, and $K$ takes an extreme value of the maximum possible value of information $I$ for this partic-ular observed phenomenon, that is

$$
K=I-J=\text { minimum }
$$


This is a principle of EPI. The extreme value is attained through a variation of the likelihood law $p(y \mid A)$ and subject to the Equation (12) connecting $I$ and $J$. The extremum is attained through a variation of the shape of $p(y \mid A)$. Equation (12) is the extremum principle used to find solution probability laws $p(y \mid A)$, and is the overall principle of "Extreme Physical Information". The naming comes from considering that the information change $I-J$ is lost information, denoted by $K$.

To understand the possibilities for instability, it is useful to reflect on the need to determine $p(x)$, thus creating a solution to the specified problem. This is subject to two aspects:

(1) Knowledge of a fixed upper-bound level $J$ to the information. All quantities are to obey a principle of minimum loss of information: $I-J=$ minimum. The $p(x)$ that obeys this minimisation principle is guaranteed to give an $I$ that is the desired maximum value. Having said this, practically, the value of $J$ assumed is no longer of interest.

(2) Knowledge of constraints that are obeyed by the unknown law $p(x)$ (as mentioned above). These are:

a. Normalisation (its integral over all $x$ equals 1 ) indicating the total probability of obtaining $x=0$ or $\Delta x$ or $2 \Delta x$ or .... (last possible value) is unity: in other words, one such value of $x$ must occur during a measurement of $x$.

b. In trying to solve for $p(x)$, one has to first go through the algebraic steps of inputting everything known about $p(x)$, such as its moments, required in order to undertake a solution procedure for $p(y \mid A)$. By "moment" is meant the average value over $x$ as a continuous integral over its range (where 1st moment is mean, 2nd moment is variance, 3rd moment is skewness). Observing fundamental effects or constraints to create inputs occurs through repeated measurements, this requiring an averaging $\langle x\rangle$.

c. Possible knowledge of correlation of $x$ with other outside variables with (say) known statistics $p(z), z$ denoting a different system. By "outside" is meant some possible other system phenomenon affecting or constraining the $x$ values that is "outside our considerations." Mathematically, this means that not enough constraints have been imposed through either: (a) that there is some other, additional set that likewise constrain the solution $p(y \mid A)$, or $(b)$ the wrong set of constraints might have been used in the first place. The constraints bound the problem specification, and change these and the problem changes.

Use of knowledge types 1 and 2 can uniquely fix the law $p(x)$. It turns out that knowledge type 1 of a particular bound level $J$ is not decisive: the solution $p(y \mid A)$, given $A$, is always independent of the size of $J$, only depending on the existence of $J$, which, in turn, follows from the philosophy of Immanuel Kant. Moreover, knowledge type 2a is not difficult to implement. However, knowledge type $2 b$ is a potential source of error. In physics, use of the least possible number of such constraints gives the best results for the analytical solution $p(y \mid A)$. In essence, the observer has to "know" what constraints are essential to the underlying effect $p(y \mid A)$. By comparison, societal or economic systems have generally more complex causes than physical ones, so it makes sense to use more constraints in analysing these by EPI.

There is an alternative way of inferring whether the ideate $\Phi$ is an adequate representation of the context represented by $A$, indicating autopoietic couple stability. This is by evaluating autopoietic efficacy $\kappa$ as indicated in Equation (10), where a high level of efficacy (as characterised by parameter value $\kappa$ ) implies coherence in the operative intelligence that defines autopoiesis. A direct way of doing this is by evaluating the General Collective Intelligence.

\section{Consequences for Metacybernetic Theory and Overview}

\subsection{Efficacy in Metacybernetics}

The above three schemas can now be used to better inform our understanding of autopoiesis in the metacybernetic framework. It has been said that metacybernetics is 
a general agency theory of higher-order cybernetics that involves intelligent complex agencies, these being dynamic systems with various self properties that enable them to adapt to changing contextual conditions. General theories have a substructure of axioms that operate with causal mechanisms through causal-agents that deliver causal effects. Here, causal mechanisms are pathways between ontologically related but distinct systems that together form the whole agency. They are populated by causal-agent networks of processes that connect these systems, and they function as agency intelligences. In a thirdorder cybernetics (as developed from Eric Schwarz [110]), a first-order causal-agent is autopoiesis (operative intelligence or self-production), and a second-order causal-agent is autogenesis (figurative intelligence or self-creation).

Both autopoiesis and autogenesis are causal-agents that generate causal effects. Anterior autopoiesis creates imperatives for regulatory change, while posterior autopoiesis creates imperatives for change in the operative structure from which agency behaviour is determined. The autopoietic couple can be seen as a cognitive system in its own right $[75,111]$. As such, this couple itself has a post-posterior strategy-regulatory system, the two being connected through autogenesis. It is here that the terminology of the anterior and posterior system comes into effect. We define a fractal structure composed of an anterior and posterior system connected through a causal-agent which has both posterior and anterior trajectories that deliver the causal effects of internalisation and anticipation. A metasystem hierarchy is composed of a sequence of embedded fractals, so that at the lowest fractal order being considered, a posterior strategic-regulatory metasystem and an anterior operative system are coupled through the causal-agent of autopoiesis. At the next fractal order up, a hierarchic level is a post-posterior metametasystem which connects with the autopoietic couple (taken as a system in its own right) through the causal-agent of autogenesis. While one may propose further hierarchies, they are beyond the scope of this paper. The strategic-regulative metasystem delivers goals, deriving from a selected cognitive purpose, which is manifested in the operative system through the causal-agent of autopoiesis as operative structure, from which behaviour arises. The strategic-regulative metametasystem has a pattern of cognitive knowledge that can be adjusted by the autopoietic couple. It also provides selected cognitive knowledge relevant to context and adaptive needs that can stimulate aspects of the autopoietic couple through the causal-agent (in this case, autogenesis), thereby influencing the strategic-regulative and operative systems. The implemented strategy stimulates adaptive operative adjustment that is constrained by a regulative structure, resulting in strategic-regulative imperatives.

We can propose that all causal-agents that connect posterior and anterior structures have both the dual cybernetic properties of internalisation and anticipation, though the meaning of these will also vary in a way that is relative to the nature of the fractal order. The causal action of autogenesis is self-production that can deliver self-organisation to either strategic-regulative structure in a higher-order posterior system (through a higher form of internalisation) or operative structure in the operative couple seen as an anterior system (through a higher form of anticipation). The causal effect of autogenesis is self-creation that can deliver either a super strategic-regulative structure to the couple by internalising super strategic-regulatory adjustments that constitute processes of learning, and through anticipation, deliver imperatives for changes to be made in the couple. While, in principle, the attributes of autopoiesis also apply to autogenesis, since both are causal-agents, our interest remains with the former.

Agency has ontologically distinct systems that interact through a network of causalagents. Agents produce or are responsible for effects that are the result of events. Examples of the distinct systems are the anterior operative system and its posterior metasystem. The operative system has a structure that creates a potential for behaviour, while the metasystem regulates/strategises the operative system and bounds/adapts behavioural potential. The metasystem and system are interconnected through the autopoietic causal-agent, a network of processes that, as already noted, can also be represented as Piaget's operative intelligence. Agencies may be any class of organism from an organisation of plural individuals to a 
solitary individual, and they may involve either implicit or explicit cognitive processes. Agency internally reflects/observes the environmental context through internalisation, this resulting in an internal cognitive model called the ideate; this, when accommodated as a structure, is now directly used in processes of self-regulation/strategization, i.e., agency uses this ideate to create requisite adaptive behaviour that responds to changing contextual conditions to enhance agency viability.

In a third-order cybernetic agency, as in Figure 1, the anterior and posterior systems are ontologically distinct, interconnected by the autopoietic causal-agent, which operates with anterior and posterior trajectories. The posterior system has cognitive schemas with the capability of creating adaptive imperatives in the anterior system. The firstorder causal-agent is autopoiesis and the second-order is autogenesis. There is also a connection between the autopoietic couple as a posterior-anterior system relationship, and its relative posterior system (the metametasystem) that is also post-posterior to the anterior (operative) system. The couple is regulated by the posterior system that has context-relative referent structures in a higher-order ideate. Examples are knowledge or identification information, or even attitudes-where implicit attitudes are those that occur with implicit cognition, and are constituted as automatic internal agency evaluations that occur without awareness. The existence of these structures is context-dependent. Sometimes, systems can become unstable due to internal contradictions. When this happens to the post-posterior metametasystem, it is no longer able to coherently regulate the autopoietic couple [17], and this can result in autopoietic couple instrumentality and instability.

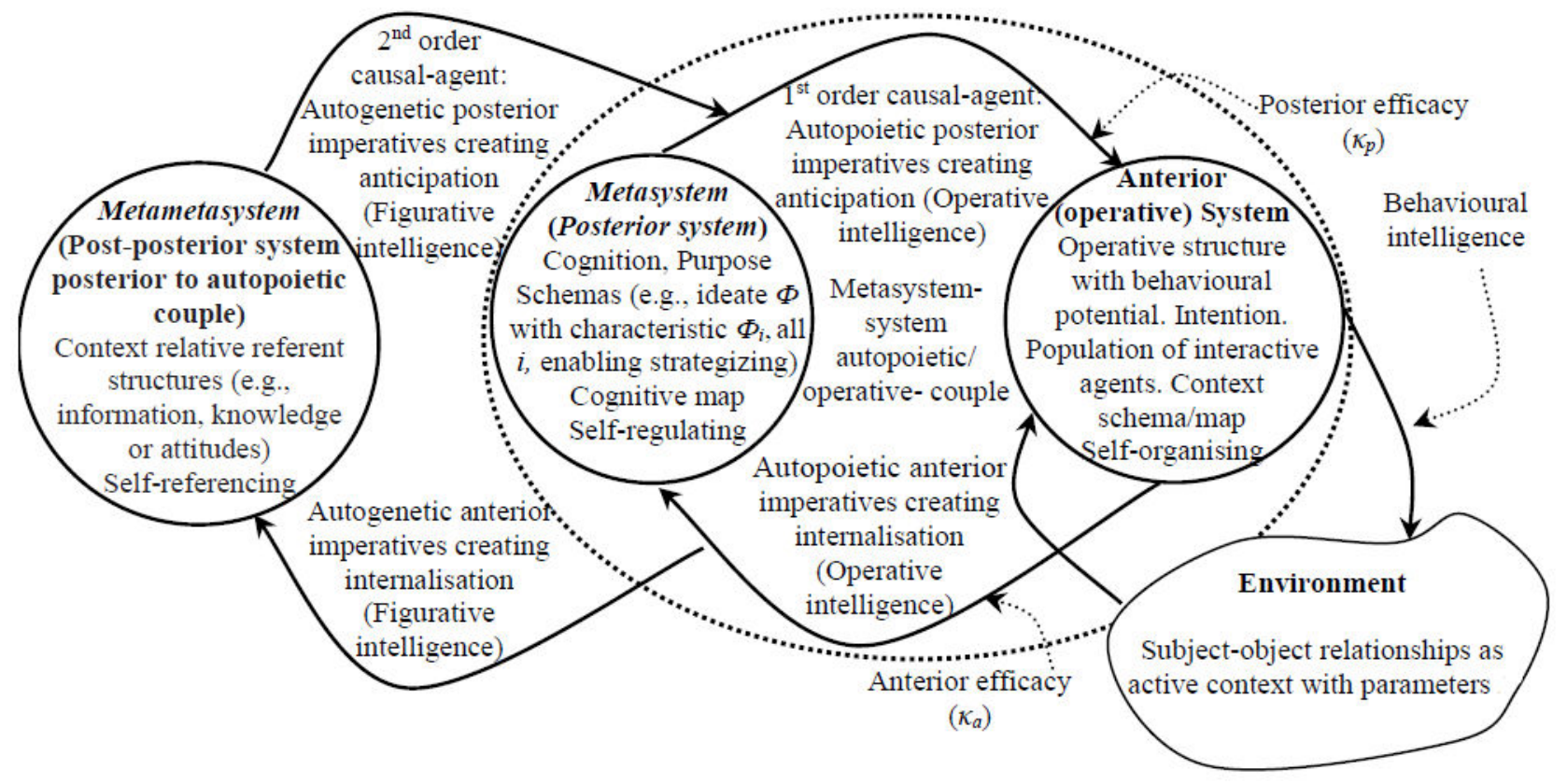

Figure 1. Third-order cybernetic agency model.

The autopoietic couple mechanisms that result in either stability or instability can be relatively easily explained in general terms using context and cognitive maps, which we shall suppose exist. Context maps represent a context with parameters $A$, together with their relationships and agent interactions, and they are connected with the structure of the anterior (operative) system. Cognitive maps represent an ideate that reflects an active context from which regulation derives. The mechanism for each autopoietic trajectory can be simply explained:

- Anterior autopoiesis acquires its notion of context from the anterior (operative) system, creates or recognises and updates the context map, and then, through internalisation, manifests a cognitive map (or cognitive map update) in the posterior system (the metasystem) as an ideate $\Phi$ (or ideate adjustment) that is assimilated and/or accom- 
modated. In the case of adjustment and with accommodation, regulative structures are modified, as may be strategic structures. In the case of autopoietic stability and with accommodation, the cognitive map is the result of a causal effect that impacts on strategic-regulative self-organisation and delivers eigenvalues.

- Posterior autopoiesis acquires its notion of the ideate from the posterior system (the metasystem), creates or recognises or updates the cognitive map, and then, through anticipation, it compares the cognitive map to the context map in the anterior system, where necessary seeking an update from behavioural intelligence (another type of causal-agent projected into active context) for the context map. This enables the operative structure to be adjusted, this modifying the potential from which behaviour results. In the case of stability and with strong anticipation, the context map is the result of a causal effect that impacts on operative self-organisation, thereby delivering eigenbehaviour.

Self-regulation is important if an agency is to be coherent, i.e., to operate in a way that enables it to have ordered behaviour in its environment through its agents. The operative regulatory structure is based on the accommodated ideate schema manifested from the context. To ensure that self-regulation is able to work successfully, the ideate must be accommodated in agency, and the autopoietic couple must be stable with respect to both anterior and posterior autopoiesis. An unstable couple can result in agency incoherency with behaviour that is entropic/disordered/uncoordinated. Disordered behaviour is not conducive to agency viability. This is especially so in a potentially malevolently changing environment in which contextual shifts demand an agency's strategic adaptation. If the ideate is not a good representation of the context, then it cannot be used to anticipate the future, and if such an ideate is accommodated into agency, it is unable to operatively self-organise suitably in a way that enables its operative adaptation to requisitely respond to the changes in context that arise from its environment. A similar argument applies in the reverse trajectory, when agency is unable to cognitively self-organise suitably in a way that enables strategic-regulative adaptation so as to requisitely respond to changes in context. Hence, anterior and posterior autopoiesis are intimately and irrevocably connected, the former delivering the causal effect of internalisation of the causal-agent anterior autopoiesis, and the latter delivering the causal effect of anticipation of the causal-agent posterior autopoiesis.

Noting that autopoiesis is operative intelligence, then there is another intelligence that represents the interaction between the anterior (operative) system and the active context, and we have already referred to this as behavioural intelligence. It relates to agency skills and abilities that arise through the agents that constitute its population, that act on its behalf or otherwise, that can contribute to behavioural anticipation and that may be responsible for delivering context maps. More specifically, behavioural intelligence allows an agency to be highly adaptable through behavioural adjustment [112] while constrained by anterior system structure (eigenbehaviour). For Yolles and Fink [113], behavioural intelligence is a form of social intelligence, which, following Thorndike [114], is the ability to understand and manage others, and to engage in adaptive social interactions.

The capability of agency to create a stable autopoietic couple is a function of autopoietic efficacy $\kappa$ (as $\kappa_{a}$ and/or $\kappa_{p}$ ). This efficacy is a result of the network of autopoietic (operative intelligence) processes working together effectively and coherently, while autopoietic couple assembly is regulated by the posterior system to this couple. Where this intelligence network is not coherent, it delivers a causal effect that is incoherent, i.e., the causal-agent observation is not well-ordered in its relation with the causal effect. In this case, the firstorder causal-agent autopoiesis can be cited as a cause for disorder/instability. Noting that there are two possible trajectories for autopoiesis, in the case of anterior autopoiesis, the ideate will, for instance, represent the context incorrectly-for instance, by biasing a set of parameters and/or parametric relationships. In the case of posterior autopoiesis, the ideate will not be well-represented as a context map from which structure and behaviour are sufficiently well-linked. 
By a recursive principle, a similar description can be applied to the relationship between the metametasystem and the autopoietic couple, where there will be an efficacy measure that reflects on the second-order causal-agent of autogenesis. It has already been noted that this couple can be seen as a cognitive system in its own right so that a causal-agent recursive principle holds, this suggesting that the term posterior system can refer to the metametasystem, and the anterior system to the autopoietic couple. Thus, in the same way that the metasystem has an ideate $\Phi$, the metametasystem may have a schema that reflects the function of the autopoietic couple. Where the metametasystem is culture, then this schema might be a knowledge structure. In this case, figurative intelligence processes will identify and select context-relevant knowledge to be used as a knowledge-accommodated higher-order ideate that will regulate the autopoietic couple.

Let us suppose that the efficacy $\mathcal{k}$ of an autopoietic process can take values between $(0,1)$ for an individual agency, where values closer to 1 indicate greater efficacy than values closer to 0 (this proposition arising from EPI). Hence, for a large $\kappa$, one may refer to autopoiesis as being efficacious. Such efficacy can be formulated in terms of the relationship between the context and the ideate. For larger values of $\kappa$, the ideate is a good representation of the context. From the work of Piaget and the Eigenform, when this occurs, we have anterior and/or posterior autopoietic stability, dependent on the orientation of the autopoietic trajectory.

Following Piaget, the autopoietic couple can be seen as stable with repeat observation (leading to information acquisition) of context by agency, thus enabling a continuity of representation of the ideate $\Phi$. Thus, stability is recognised with an indefinite succession of recursive cognitive operations ([19,88]. We can note Piaget's [88] explanation that an observer observes the developing interaction between a subject and an object [115]. This relationship is recursive in the process of observation, and it connects the observer with the subject-object operation that is formed as a coupled structure, continually refreshed with respect to previous observations. It thus defines a repetitive cognitive process that occurs through internalisation, emerging as a symbolic cognitive elaboration in the subject-object relationship, where the operation is symbolically enhanced through cognitive processes. Essentially, recursive processes centre on autopoietic imperatives that self-produce agency change, and that arise from the anterior (operative) system. In contrast, Dubois' distinction between recursive and incursive processes highlights the autopoietic processes at work that create anticipation, this defining agency as a living system with an autonomous adaptive capacity.

It has been said that context is both complex and dynamic, and, consequently, it is time-dependent, as, therefore, will be the ideate $\Phi$ that is manifested from that context.

Consider now that the autopoietic couple delivers the ideate $\Phi$ deriving through autopoiesis and representing a contextual parameter $A$. The ideate $\Phi$ is therefore an autopoietic function of the qualitative and / or quantitative parameters $A$, where quantitative parameters might, for instance, be expressed as boundaries that differentiate selectable qualities that may have statistical properties [44].

We have earlier noted that the process of internalisation includes assimilation (when $A$ is introduced as a model into the posterior system/metasystem, which agency then uses to weakly anticipate behaviour), and accommodated (as a posterior system schematic structure, which agency then uses to strongly anticipate behaviours). If, after accommodation, the characteristics of the ideate $\Phi$ are insufficiently close to those of the context, then agency is unable to realistically anticipate behaviour, i.e., behaviour will not correspond to agency requisite needs. In this case, the operative couple is unstable. Stability is required if, under complexity, agency is able to behave in a requisite way such that any potentially malevolent environmental influence can be responded to suitably, so as to ensure its viability. This is dependent on autopoietic stability, so that the coherent operative structure (in the operative system), from which behaviour derives, can be self-produced. The autopoietic couple is serviced by the autopoietic network of processes that constitutes agency operative intelligence. This is intimately involved in the reflecting task of observation, 
where operative intelligence identifies and selects context-representative parameters, and then processes them to assimilate the model using a cognitive map followed, if feasible, by accommodation.

Since metacybernetics embraces critical realism, it should consider both qualitative and quantitative approaches to inquiry. Thus far, in metacybernetics, we have considered only qualitative aspects that are essentially constructivist, and perhaps it is time to turn our attention to quantitative aspects in which numerical data are used to obtain information about the world [116]. Now, metacybernetics formulated as a quantitative theory is an ally of EPI, this we recall being a measurement centred on general information theory that allows $A$ to be ultimately found, given prior knowledge of constraints such as mean value or variance. As a probabilistic approach, we recall that it assumes that context can be represented by the unknown law $p(x)$ under the condition from the EPI principle that acquired information is maximised.

This can be explored further in relation to contexts. Consider that the ideate is a token used by agency to interact with other attributes in a context. Contexts can deliver complex problems that may be wicked. We note that complex problems have tangled structures and processes. Tight tangles deliver wicked problems, where there is insufficient knowledge to adequately define the problem or create relevant intervention strategies. Where relevant interventions are deemed to occur, they have effects that change the problem, thus making it self-perpetuating [115]. There are additionally hidden attributes so that relationships remain unclear, and wicked problems are effectively unresolvable and the idea of optimisation unrealistic [117]. Thus, especially in wicked problems, there is a need to make more than one observation if one wishes to ascertain whether and how the characteristics represented by a predetermined contextual parameter have changed.

Let us now turn our attention to anterior autopoiesis, noting that EPI is concerned with the measurement of context. Essentially, an agency observing a context over time internalises it to form an ideate. The anterior autopoietic relationship is stable if the ideate sufficiently well represents context. In terms of information theory, conceptually, the manifestation of the ideate arises because information has been acquired from the context. The context may be represented through an observation (or, more correctly, a measure) $y$, and, as indicated in Equation (7), it essentially derives from the parameters (providing meaning). As such, the observation is indicative of the nature of a context only if one takes into account any observational failing indicated by some random variable $x$, and that will be related to the noise that might arise in the anterior (operative) system context map, and then autopoietically manifested in the posterior system ideate $\Phi$. To determine autopoietic couple stability iteratively, and thus decide whether $\Phi$ is an adequate representation of a context, one can take recurrent observations from which arise the set of parameters $A$ in Equation (7). In this case, the need is to compare the set of data $y(t)$. To determine stability, one is required to evaluate the variance of the probability set $p(y(t) / A)$, which brings us to consider its composition. It embeds a set of parameters $A$ that are the contextual descriptors, and those that are statistically measurable will have mean, variance, and perhaps bias associated with it (cf. [118]).

\subsection{Overview and Discussion}

At this juncture, it will be useful to take an overview of the nature of agency theory within metacybernetics, where agencies are taken as living systems that have a variety of self properties, and they maintain their viability through changing contexts and adaptation. To adapt, they need to internalise the active context that they observe, and to anticipate the future so that their behaviours are requisite. The two properties associated with internalisation are assimilation and accommodation, and agencies that only assimilate have different anticipatory properties from those also able to (cognitively) accommodate an observed context, this impacting on the nature of living.

Internalisation occurs through autopoiesis, a topic that is of paramount importance when considering agency capacity to seek viability. Anticipation also occurs through 
autopoiesis, and is a notion that is required if agency is deemed to be living. This suggests that there are reverse trajectories for autopoiesis, anterior and posterior, both of which are capable of delivering imperatives for structural change. The anterior trajectory provides an imperative that derives from anterior autopoiesis and impacts on strategy-regulatory structures. The posterior trajectory provides an imperative that derives from posterior autopoiesis that is directed towards possible agency operative structure adjustment. As such, agencies are able to self-organise both anteriorly and posteriorly, an idea that redefines by extension the normal understanding of self-organisation. In a lesser situation than that provided, posterior autopoiesis might be considered to be equivalent to feedforth from a system to its metasystem, while anterior autopoiesis has equivalence with feedback. However, such terms are inadequate to describe the processes that take place in general terms, especially with higher-order cybernetic systems. It may also be noted that anterior and posterior autopoietic processes should, in general, be considered to be autonomous and interactive, though their relationship is likely to be non-linear in time and space.

While it is usual to talk about self-organisation only in terms of the development of an anterior (operative) system structure, it is clear that its strategic and regulative functionalities (essentially a posterior cognitive attribute) are also structured. Consequently, any self-change in these cognitive structures is also necessarily a result of self-organisation. To appreciate the consequences of this, a proper understanding of the concept of autopoiesis is required, and which we improved. This has been due to the consideration of the three schemas described. There is also an issue concerning the qualities that determine autopoietic efficacy that have arisen in Section 3 of this paper.

For David [119], autopoiesis has been a mainly theoretical concept, but it can be used to generate pragmatic outcomes. In this spirit, we have discussed the qualitative nature of autopoietic efficacy and explored a more generalised quantitative measure for it. Efficacy may be defined as agency capability in producing a treatment that, if applied ideally, can result in beneficial effects in real-world settings. By treatment is meant the action or manner (as promoted by a particular schema) of dealing with something, and where the successful application of the schema will ideally satisfy intended agency needs [120]. What capability refers to will vary with the framework used, i.e., the nature of efficacy varies as a function of the paradigm from which a particular schema arises. By schema is meant a structured knowledge framework which defines a pattern of thought/behaviour, and which has an organisation of information categories and effect relationships [121]. They have propositions about their characteristics, relationships, and entailments, may operate with incomplete information, and may refer to simple abstract concepts or complex social phenomena. Here, three schemas have been identified and have effectively been filtered through a metacybernetic framework to enable them to be mutually configured with reference to autopoiesis. Metacybernetics [17] is a general theory that is able to configure specific theoretical approaches to respond to particular contexts, where a context is described by information that characterises a situation through a set of parameters [122].

A plurality of commensurable schemas may be configured, where each has inherent coordinative structures able to respond to the needs of specific contextual modelling. A plurality of configurations operates as a complex system of interdependencies, therefore having core orchestrating themes with identifiable characteristics. It should be noted that metacybernetics is a critical realism paradigm. This involves inquiry into reality through both a constructivist-qualitative approach centring on cognition, and a realist-quantitative approach that centres on measurement. Both have relevance to understanding autopoietic efficacy. Metacybernetics, as a general theory, has a superstructure that builds theory through configurations, and a substructure of axiomatic principles involving causal-agents (entities that produce effects that are an inevitable result of a causal antecedent). Efficacy is determined through an evaluated causal effect of treatments which arise from a causalagent, and it occurs when the effect is requisite - that is, made necessary for viability by particular circumstances. 
Here, in discussing the nature of autopoiesis, we formulated some propositions that derived from the three schemas introduced, set up within centripetal arguments that enabled recognition of common commensurability. We harnessed the schemas by exploring aspects of autopoietic efficacy relative to their paradigmatic perspectives. GCI comes from knowledge management, and, as discussed by Krafft [71], concerns group performance across correlated tasks, and it is a correlation measure that is an indicator of collective intelligence [123]. Adopting a centripetal argument that imputes/indicates autopoiesis, it was explained how GCI is a measure of autopoietic efficacy. Coming from the theory of knowledge, we reformulated Eigenform [95] as a dual cybernetic theory. Composed of two parts, the first arose from Foerster [19], which considers observation through which a process of internalisation occurs, where an observer and an observed context are connected, and which uses recursive logic to explore this. The second came from Dubois [98], who considers anticipation through incursive logic. Foerster's approach informs that of Dubois, and Eigenform therefore provides the validity required to support the propositions that anterior and posterior autopoiesis occur as reverse trajectories. The third approach considered was that of EPI, an information theory that centres on the observation of active contexts, this able to identify conditions for efficacy given parameters that describe the context under uncertainty.

Now, Eigenform and EPI are connected. Eigenform (in its extended form) has interests in the logical process associated with anterior and posterior autopoietic trajectories. EPI, as represented here, is concerned with observed contexts through anterior sampling resulting in the internalisation of information flows. GCI, however, is concerned with group performance across correlated tasks, and it needs to be explained how this may be related to observation and autopoiesis. The need, then, was to examine their features through centripetal discourse to enable autopoiesis to emerge as a linkage between them.

Agencies are adaptive living systems that can internalise an observed context, and create an ideate that it can use to anticipate its behaviour. Both internalisation and anticipation are intimately and irrevocably connected, the former a causal effect of the causal-agent of anterior autopoiesis, and the latter a causal effect of the causal-agent of posterior autopoiesis. Internalisation is a function of anterior autopoiesis, with two states:

- The first state of internalisation occurs through anterior autopoiesis, which involves an autopoietically constructed model of an observed and recognised active context that the agency assimilates internally as an ideate, and which delivers only implicit cognition and recognition, both of which are information processes.

- The second state of internalisation increases complexification, incorporating the assimilated model into the agency posterior system structure through accommodation, this leading to explicit cognition and recognition, enabling conscious awareness with rationality.

Anticipation is a function of posterior autopoiesis, and has two states:

- The first state of anticipation is a consequence of assimilation, and corresponds to exoanticipation, where agency anticipates the external active context through expectations about the need for future behaviour.

- The second state of anticipation is a consequence of accommodation, and corresponds to endo-anticipation, when the ideate becomes embedded in the agency structure, which then determines its eigenbehaviour (as operative structure) and creates potential for future behaviour.

Autopoietic efficacy is an indicator of the capability of autopoiesis to deliver requisite effects. In exploring autopoietic efficacy through the three schemas, it must be recognised that GCI did not originate as an autopoietic schema, but here, our centripetal arguments, independently supported by Halpin [25], have enabled autopoiesis to be defined as a pragmatic set of contextually connected purposeful and relatable tasks, each undertaken by a subagency as a team of processing agents that are members of agency population. Efficacy, a capability to create a beneficial effect in real-world settings (such as effectively 
manifesting goals that are sustainable), is sought for the whole assembly. Eigenform is a logical symbolic approach and, from our centripetal argument, it is shown that autopoiesis requires stability if agency is to operate coherently-in other words, when autopoietic efficacy may be seen in terms of anterior autopoietic stability delivering eigenvalues, and when posterior autopoiesis stability delivers eigenbehaviours with a potential for requisite behaviour. In essence, then, Eigenform is concerned with how observations can be suitably internalised and thus be used as a token to determine future behaviour. Dubois' elaboration of Foerster's ideas (of internalisation that, at its very best, implies anticipation) extended Eigenform theory to an explicit consideration of anticipation. EPI is a schema, the mechanisms of which constitute an autopoietic process that is concerned with gleaning the nature of a context from the observation of samples from it through parameters that are able to describe and give meaning to it.

The configurative relationship between the three schemas can be summarised as follows. GCI seeks, for the whole network of autopoietic processes, a collective intelligence, the efficacy of which may be measured through correlation. Since correlation may be expressed in terms of joint probabilities, it has immediate connections with EPI, the interest of which centres (within the theme of this paper) on identifying a measure of autopoietic efficacy from observation of an active context through measurement sampling, and where hidden parameters might be inferred from probability distributions, given prior knowledge. This provides opportunities for GCI and EPI to function together. Eigenform is a symbolic approach that creates an argument for autopoietic couple stability, this underpinning both GCI and EPI in their agency application. Foerster's approach centres on observation, this delivering the implicit recognition of a developing context through repeat observations in situations that suppose coherent dynamic continuity. It was formulated for explicit cognition, where agencies show conscious awareness, though it is relevant to agencies with implicit cognition. Foerster's approach centred on anterior autopoiesis (internalisation), where stability delivers eigenvalues. Stability, he indicated, then applies to agency as a whole. However, breaking agency down into anterior and posterior forms of autopoiesis suggests a different proposition. It is that anterior and posterior autopoiesis both have their individual dynamics. In the case of the former, stability delivers eigenvalues, and in the latter case, stability delivers eigenbehaviour as an agency operative structure, which in turn creates potential for behaviour. Clearly, though, eigenbehaviour is dependent on the existence of eigenvalues. One may contrast Eigenform with GCI and EPI, the latter pair also inherently allowing for implicit cognition. This does not require any awareness assumptions, but does require that there is an active information process that enables self properties. Thus, for instance, in the case of GCI, which is designed for intelligent aware organisations, their collective intelligence (which is underpinned by patterns of norms, assumptions, and beliefs prevalent in even a distributed culture) does not necessarily imply a collective conscious awareness that enables agency as the collective whole to assume explicit cognition [124]. Hence, in terms of the Bitbol and Luisi [6] propositions about stages of consciousness, GCI refers to stage 4 cognition that seeks evolutionary development into stage 5 .

The connection between metacybernetics and EPI has been explored elsewhere [23], and GCI had the potential to be configured into this. Through metacybernetics, GCI and EPI therefore can, in principle, act as mutual verifiers for relevant situations, since they can each independently obtain a measure of autopoietic efficacy. GCI has a developing approach using multi-agent techniques through which algorithms are developed to determine measures. However, these are still subject to hidden parameters and dynamics. It is feasible, then, for GCI to incorporate EPI, enabling it to seek hidden parameter inferences.

Eigenform and GCI are specific theories that sit comfortably within a second-order cybernetics and its associated autopoiesis. EPI, however, as a general theory, is, in principle, able to generate measurement criteria for higher-order cybernetic structures through metacybernetics that conform to post-nonclassical rationality. It has been noted that Eigenform provides a logical basis for the conditions that prevail for autopoietic stability by 
exploring recursive operations of observation, but clearly, as a logical structure, it offers no pragmatic orientation.

The Eigenform approach arises from the individual studies of Foerster, Rocha, and Kauffman. It formulates a recursive expression for observation, the solution of which implies autopoietic couple stability that in turn implies autopoietic efficacy. Kauffman applied Spencer Brown's logical structures to Foerster's approach, resulting in the view in cybernetics that these are underpinning attributes for the idea of observation and, indeed, autopoiesis. In Foerster's original development, he produced the notion of the eigenvalue, which exists only with stability, and eigenbehaviour that requires the existence of an eigenvalue, and supports stable self-production and which in turn creates the potential for stable behaviour, though how this may occur is really a mystery since it is not explained. The elaboration of the recursive notions of Foerster to include incursive notions as provided by Dubois extends the notion of Eigenform to specifically embrace anticipation, significant in that it simply defines living. In the same way that Foerster's recursive equations, when solved, imply stability, so too do those of Dubois.

We have argued that GCI is closely linked to autopoiesis and autopoietic efficacy, and we have already noted that this is a notion independently supported by Halpin. The efficacy value can be determined by EPI from a probability distribution that measures acquired information $I$, where a large value of $I$ is an indicator of order and implies autopoietic couple stability, at least with respect to anterior autopoiesis. EPI is probabilistic, developing from propositions of Fisher information to respond to issues of uncertainty. When it uses the term observation, it is referring to explicit observation, meaning that it extrinsically recognises and notes attributes of context acquired through measurement. This measurement is a single observation of context, given pre-knowledge of Gaussian measures such as mean and variance.

\section{Conclusions}

The purpose of this paper has been to explore the nature of autopoiesis and its efficacy within the context of living systems, and in doing this, we have adopted three independent, demonstrably commensurable schemas. These schemas have been explored in terms of their interests, showing that they are relatable, and through centripetal arguments, thereby explaining how they are each related through autopoiesis, their properties, then providing greater insights into the nature of autopoietic processes.

To summarise this development within an agency context, agencies are here taken to be living systems through their capacity to anticipate, this being dependent on its capability for internalisation. This applies to all forms of living system, whether they are biological, chemical, psychological, mechanical, or social. There are qualitative grades of agency competence, this being explained in terms of their cognitive ability enabling them to have a capacity for recognition. There are two types of cognition, implicit and explicit. Implicit cognition occurs in a lesser form of life that involves an automatic information-based process that operates rapidly with less information than explicit cognition, which is a more complex form of life that involves rational processes. Agencies may also be conscious entities. Consciousness may start as a primitive condition, and then develop. Primitive consciousness is connected with agency learning and an ability to modify configurations and states in adaptation, enabling evolution and self-regulation. Developmental consciousness involves an evolutionary agency process (since evolution is a collective process, and agency is constituted through a population of agents) based on stable dynamic support yielding to strongly anticipative behaviour, with enhancing memory structures and increasingly developing regulatory and adaptive attributes.

Having referred to recognition, agencies observe contexts when they are recognising and noting parametric attributes of context. Implicit recognition occurs where the discrimination of context involves repeated exposure to memory through information processing. Explicit recognition is more complex and involves the conscious discrimination of parametric attributes, and is associated with rapid perceptual learning in identifying context. 
Agencies operate through causal mechanisms populated by causal-agents that deliver causal effects. Autopoiesis (as with autogenesis) is a causal-agent that has a dual cybernetic functionality through its reverse trajectories. These produce the different causal effects of internalisation and anticipation. Internalisation arises as an effect through anterior autopoiesis, while anticipation arises as an effect from posterior autopoiesis. That these two causal-agents exist extends traditional autopoietic theory, not only with respect to the introduction of dual reverse trajectories, but also in redefining the notion of self-organisation so that it applies not only, as in tradition, to the anterior structure from which behaviour emanates, but also to the strategic-regulative structure that ultimately creates opportunities for adaptive behaviour and bounds on what behaviour is permissible. Anterior autopoiesis is responsible for agency regulative capacity, while posterior autopoiesis is responsible for operative structure and hence behaviour. Anterior and posterior autopoiesis connect an operative system with its metasystem in a first-order fractal (in an embedded sequence of higher order fractals in a metasystem hierarchy), producing an autopoietic couple. This couple may be seen as a system in its own right, which itself may be regulated by a higher-order strategic regulator, perhaps called a metametasystem (a post-posterior system relative to agency's anterior system). This applies to higher cybernetic orders that have causal-agents (similar to autogenesis) delivering causal effects. These causal effects are always internalisation and anticipation, but the nature of these effects alters with the ontological context, i.e., related to the nature of the cybernetic order being considered. These relationships are reflected in the poem "Siphonaptera," by Augustus De Morgan, in which "great fleas have little fleas upon their backs to bite 'em, and little fleas have lesser fleas, and so ad infinitum."

Both anterior and posterior autopoiesis can be measured. Two approaches have been identified for this, one coming from GCI, and the other from EPI. The efficacy measure for the internalisation causal effect is that of anterior autopoiesis represented by $\kappa_{a}$, and that for the anticipation causal effect is that of posterior autopoiesis represented by $\kappa_{p}$. Since, according to GCI, the subagencies that are involved in each trajectory are autonomous and organisationally distributed, there is no reason to suppose that $\kappa_{a}=\kappa_{p}$, and likely they will normally be different. The Dubois Eigenform equations that represent these two causal-agent processes, when solvable, indicate autopoietic stability. During internalisation, stability results in eigenvalues, and during anticipation, stability results in eigenbehaviour. It should be realised that eigenbehaviour is nothing other than the operative structure that enables the potential for behaviour. Thus, the teeth of a lion or the neck of a giraffe are operative structures that directly influence behaviours.

Now, an autopoietic couple is a second-order cybernetic system with a first-order causal-agent called autopoiesis (self-production), and a third-order cybernetic system operates in exactly the same way, except that its second-order causal-agent is now called autogenesis (self-creation). While self-production is an effect that can apply to both strategic regulation and operative structure from which behaviour arises, self-creation is rather concerned with learning/innovation and strategic behaviour adjustment. The core cybernetic model involving the causal-agents that define an anterior and posterior couple is therefore recursive.

When considering internalisation, we are referring to context, defined as the interrelated conditions in which something exists or occurs, including objects, subjects, their interactions, and the resulting effects. Traditionally, a second-order system is considered to be an observing system. This implies that it is the posterior system that "looks on" at a context thereby observing it. However, this is a proposition that, if correctly represented, would seem false. Rather, observation is due to behavioural intelligence from which that being observed is manifested as a context map, and then through autopoiesis manifested as a cognitive map from which an ideate emerges in that posterior system. This is so even while the posterior system is a regulator of agency as a whole.

Observation of an active context is represented in the anterior (operative) system and becomes manifested as an ideate image in its posterior system (e.g., the metasystem). 
Occurring through internalisation as an effect, it has two possible states. One is assimilation, which is information-based, and the other accommodation, which is structure-based. Agency observation may also be of the ideate that is connected with regulation, occurring through posterior autopoiesis, when the causal effect is anticipation. This is the alternative to internalisation with a reverse trajectory, and which also has two states, weak and strong. Assimilation is tied to weak anticipation delivering an agency with lesser complexification, while accommodation is tied to strong anticipation and delivers an agency with greater complexification.

These propositions are the result of considering the three schemas that we have introduced within the metacybernetic framework. They lead to stark distinctions from more traditional cybernetic theory that mostly centres on second-order cybernetics, and creates mystification around eigenvalues and eigenbehaviours. It thus provides an evolutionary shift in the development of metacybernetics and the causal processes (of which autopoiesis is one) that define the nature of living.

Author Contributions: M.Y. is the primary conceptual author, with a specialism in social cybernetics and, in particular, in the general theory of metacybermetics, which he originated. B.R.F. has provided conceptual support with respect to the mathematical structures provided and their elaboration, especially with respect to EPI, a general theory that originates with him. All authors have read and agreed to the published version of the manuscript.

Funding: This research received no external funding.

Conflicts of Interest: The authors declare no conflict of interest.

\section{References}

1. Maturana, H. Biology of Cognition; Biological Computer Laboratory Research Report BCL 9.0; University of Illinois: Urbana, IL, USA, 1970.

2. Farnsworth, K.D.; Nelson, J.; Gershenson, C. Living is Information Processing: From Molecules to Global Systems. Acta Biotheor. 2013, 61, 203-222. [CrossRef]

3. Simon, H. Cognitive Science: The Newest Science of the Artificial. Cogn. Sci. 1980, 4, 33-46. [CrossRef]

4. Griffin, D.R. From cognition to consciousness. Anim. Cogn. 1998, 1, 3-16. [CrossRef]

5. Bensaude-Vincent, B. Self-Assembly, Self-Organization: A Philosophical Perspective on a Major Challenge of Nanotechnology. In Proceedings of the France-Stanford Meeting Implications Sociales et Ethiquesde la Convergence Nano-, Bio-, Info- Cogno-, Avignon, France, 17 December 2006.

6. Bitbol, M.; Luisi, P.L. Autopoiesis with or without cognition: Defining life at its edge. J. R. Soc. Interface 2004, 1, 99-107. [CrossRef]

7. Yolles, M. Agency, ecosystems and sustainable development. Part 1: The ecosystem. Kybernetes 2021. [CrossRef]

8. Bielecki, A. The Systemic Concept of Contextual Truth. Found Sci. 2020. [CrossRef]

9. Korzeniewski, B. Cybernetic formulation of the definition of life. J. Theor. Biol. 2001, 209, 275-286. [CrossRef] [PubMed]

10. Simon, H.A. The architecture of complexity. In Facets of Systems Science; Springer: Boston, MA, USA, $1991 ;$ pp. 457-476.

11. Wiener, N. Cybernetics or Control and Communication in the Animal and the Machine; Wiley: New York, NY, USA, 1961.

12. Rosen, R. Anticipatory Systems; Pergamon Press: New York, NY, USA, 1985.

13. Maturana, H.R.; Varela, F. Autopoiesis and Cognition: The Realization of the Living; Reidel: Boston, MA, USA, 1980.

14. Hornung, B. Sociocybernetics. In International Encyclopedia of the Social and Behavioral Sciences, 2nd ed.; Elsevier: Amsterdam, The Netherlands, 2015; pp. 912-917.

15. Razeto-Barry, P. Autopoiesis 40 years later. A review and a reformulation. Orig. Life Evol. Biosph. 2012, 42, 543-567. [CrossRef]

16. Li, Q.; Clark, B.; Winchester, I. Instructional design and technology grounded in enactivism: A paradigm shift? Br. J. Educ. Technol. 2010, 41, 403-419. [CrossRef]

17. Yolles, M. Metacybernetics: Towards a General Theory of Higher Order Cybernetics. Systems 2021, 9, 34. [CrossRef]

18. Dopfer, K.; Foster, J.; Potts, J. Micro-meso-macro. J. Evol. Econ. 2004, 14, 263-279. [CrossRef]

19. Foerster, V.H. Objects: Tokens for (Eigen)-Behaviors. Cybern. Forum 1976, 8, 91-96.

20. Dubois, D.M. Review of incursive, hyperincursive and anticipatory systems-foundation of anticipation in electromagnetism. AIP Conf. Proc. 2000, 517, 3-30. [CrossRef]

21. Brown, T. Making Truth: Metaphor in Science; University of Illinois Press: Champaign, IL, USA, 2003

22. Lakoff, G.; Johnson, M. Metaphors We Live By; University of Chicago Press: Chicago, IL, USA, 1980.

23. Yolles, M.I.; Fink, G. A Configuration Approach to Mindset Agency Theory-A Formative Trait Psychology with Affect Conition and Behaviour; Cambridge University Press: Cambridge, UK, 2021.

24. Miller, D. Configurations revisited. Strateg. Manag. J. 1996, 17, 505-512. [CrossRef] 
25. Halpin, H. Foundations of a Philosophy of Collective Intelligence. In Proceedings of the AISB Convention, Birmingham, UK, 2-6 July 2012.

26. Bandura, A. Human agency in social cognitive theory. Am. Psychol. 1989, 44, 1175-1184. [CrossRef]

27. Kauffman, L. Eigenform. In Proceedings of the 51st Annual Meeting of the ISSS, Tokyo, Japan, 5-10 August 2007.

28. Wegener, R. Parameters of Context: From Theory to Model and Application. Ph.D. Thesis, Macquarie University, Sydney, Australia, 2011.

29. Steele, A.; Leitch, R.R. Qualitative Parameter Identification. In Proceedings of the QR-97 11th International Workshop on Qualitative Reasoning about Physical Systems, Cortona, Italy, 3-6 June 1997.

30. Brooks, S.; Gelman, A. Some issues for monitoring convergence of iterative simulations. In Proceedings of the Section on Statistical Computing ASA, Washington, DC, USA, 13-16 August 1998.

31. Yolles, M.I. Least Squares Successive Relaxation. IMA J. Appl. Math. 1975, 16, 329-343. [CrossRef]

32. Beaufils, B.; Delahaye, J.P.; Mathieu, P. Our meeting with gradual, a good strategy for the iterated prisoner's dilemma. In Proceedings of the Fifth International Workshop on the Synthesis and Simulation of Living Systems, Nara, Japan, 16-18 May 1996.

33. Leinonen, A.; Rasilainen, K.; Komonen, P.; Gotcheva, N. Nuclear Waste Repository as a Scenario Problem: Developing Epistemic Understanding; VTT Technical Research Centre of Finland: Espoo, Finland, 2021.

34. Tietje, O. Identification of a small reliable and efficient set of consistent scenarios. Eur. J. Oper. Res. 2005, 162, 418-432. [CrossRef]

35. Chakir, R.; Lungarskay, A. Agricultural land rents in land use models: A spatial econometric analysis. In Proceedings of the 150th Seminar (EAAE) European Association of Agricultural Economists, Edinburgh, UK, 22-23 October 2015.

36. Spathopoulou, F.; Papakonstantinidis, S. Culture as a parameter in assessing students performance. Humanit. Soc. Sci. Lett. 2017, 5, 72-78.

37. Parrish, P.; Linder-VanBerschot, J. Cultural dimensions of learning: Addressing the challenges of multicultural instruction. Int. Rev. Res. Open Distrib. Learn. 2010, 11, 1-19. [CrossRef]

38. Hofstede, G.; Hofstede, G.J. Cultures and Organizations: Software of the Mind; McGraw-Hill: New York, NY, USA, 2005.

39. Nisbett, R.E. The Geography of Thought: How Asians and Westerners Think Differently - And Why; Free Press: New York, NY, USA, 2003.

40. Levine, R. A Geography of Time; Basic Books: New York, NY, USA, 1997.

41. Hall, E.T. Beyond Culture; Random House: New York, NY, USA, 1981.

42. Lewis, R.D. When Cultures Collide: Leading Across Cultures; Nicholas Brealey International: Boston, MA, USA, 2006.

43. Tamis-LeMonda, C.S.; Way, N.; Hughes, D.; Yoshikawa, H.; Kalman, R.K.; Niwa, E.Y. Parents' Goals for Children: The Dynamic Coexistence of Individualism and Collectivism in Cultures and Individuals. Soc. Dev. 2007, 17, 183-209. [CrossRef]

44. Yolles, M. Changing paradigms in operational research. Cybern. Syst. 1998, 29, 91-112. [CrossRef]

45. Ha, S.; Jeong, H. Unraveling hidden interactions in complex systems with deep learning. Sci. Rep. 2021, 11, 1-13. [CrossRef] [PubMed]

46. Glanzberg, M. Not All Contextual Parameters Are Alike; University of Oslo: Oslo, Norway, 2009.

47. Stojanovic, I. Emotional Disagreement: The Role of Semantic Content in the Expression of, and Disagreement Over, Emotional Values. Dialogue Can. Philos. Rev. 2012, 51, 99-117. [CrossRef]

48. Foerster, V.H. Cybernetics of Cybernetics. In Communication and Control in Society; Gordon and Breach: New York, NY, USA, 1979.

49. Tolman, E.C. Cognitive maps in rats and men. Psychol. Rev. 1948, 55, 189-208. [CrossRef] [PubMed]

50. Heft, H. Environment, cognition, and culture: Reconsidering the cognitive map. J. Environ. Psychol. 2013, 33, 14-25. [CrossRef]

51. Kitchin, R.M. Cognitive maps: What are they and why study them? J. Environ. Psychol. 1994, 14, 1-19. [CrossRef]

52. Russell, B. Vagueness. Australas. J. Psychol. Philos. 1923, 1, 84-92. [CrossRef]

53. Yolles, M. From Competitive Advantage to Knowledge Cybernetics. Syst. Integr. 2006, 72. Available online: http:// citeseerx.ist. psu.edu/viewdoc/download?doi=10.1.1.491.1609\&rep=rep1\&type=pdf (accessed on 22 October 2021).

54. Henderson, J.M. Regarding scenes. Curr. Dir. Psychol. Sci. 2007, 16, 219-222. [CrossRef]

55. Cao, Y. An Information Assistant System for the Prevention of Tunnel Vision in Crisis Management; University of Twente: Twente, The Netherlands, 2008.

56. Underwood, G.; Bright, J.E.H. Cognition with and Without Awareness; Oxford University Press: Oxford, UK, 1995.

57. Nosek, B.A. Implicit-Explicit Relations. Curr. Dir. Psychol. Sci. 2007, 16, 65-69. [CrossRef]

58. Nosek, B.A.; Hawkins, C.B.; Frazier, R.S. Implicit social cognition: From measures to mechanisms. Trends Cogn. Sci. 2011, 15, 152-159. [CrossRef]

59. Stammers, S. Is Implicit Cognition Bad Cognition? 2016. Available online: https://icog.group.shef.ac.uk/is-implicit-cognitionbad-cognition (accessed on 22 October 2021).

60. Cunningham, W.A.; Zelazo, P.D.; Packer, D.J.; Van Bavel, J.J. The Iterative Reprocessing Model: A Multilevel Framework for Attitudes and Evaluation. Soc. Cogn. 2007, 25, 736-760. [CrossRef]

61. de Lemos, R.; Grześ, M. Self-adaptive artificial intelligence. In Proceedings of the IEEE/ACM 14th International Symposium on Software Engineering for Adaptive and Self-Managing Systems (SEAMS), Montreal, QC, Canada, 25 May 2019.

62. Chella, A.; Pipitone, A.; Morin, A.; Racy, F. Developing Self-Awareness in Robots via Inner Speech. Front. Robot. AI 2020, 7. [CrossRef] 
63. Chatila, R.; Renaudo, E.; Andries, M.; Chavez-Garcia, R.-O.; Luce-Vayrac, P.; Gottstein, R.; Alami, R.; Clodic, A.; Devin, S.; Girard, B.; et al. Toward Self-Aware Robots. Front. Robot. AI 2018, 5. [CrossRef] [PubMed]

64. Prentner, R. Consciousness: A Molecular Perspective. Philosophies 2017, 2, 26. [CrossRef]

65. Kinouchi, Y.; Kato, Y. A Model of Primitive Consciousness Based on System-Level Learning Activity in Autonomous Adaptation. In Biologically Inspired Cognitive Architectures 2012; Springer: Berlin/Heidelberg, Germany, 2013.

66. Lehn, J.-M. Supramolecular chemistry? Scope and perspectives: Molecules? Supermolecules? Molecular devices. J. Incl. Phenom. Macrocycl. Chem. 1988, 6, 351-396. [CrossRef]

67. Carr, H.W. The problem of recognition. Proc. Aristot. Soc. 1916, 17, 1-27. [CrossRef]

68. Thibierge, S.; Morin, C. Which Identification is Disturbed in Misidentification Syndromes? A Structural Analysis of Fregoli and Capgras Syndromes. J. Mind Behav. 2016, 37, 1-13.

69. Martens, U.; Wahl, P.; Hassler, U.; Friese, U.; Gruber, T. Implicit and Explicit Contributions to Object Recognition: Evidence from Rapid Perceptual Learning. PLoS ONE 2012, 7, e47009. [CrossRef]

70. Lehn, J.-M. Toward complex matter: Supramolecular chemistry and self-organization. Proc. Natl. Acad. Sci. USA 2002, 99, 4763-4768. [CrossRef] [PubMed]

71. Krafft, P.M. A Simple Computational Theory of General Collective Intelligence. Top. Cogn. Sci. 2018, 11, 374-392. [CrossRef]

72. Williams, A. Functional Modeling and General Collective Intelligence as the Basis for Pervasive Healthcare. arXiv 2021, arXiv:africarxiv/4wzft. Available online: https://osf.io/preprints/africarxiv /4wzft (accessed on 22 October 2021).

73. Williams, A.E. Applications for General Collective Intelligence. arXiv 2020. Available online: https:/ / osf.io/preprints/africarxiv/ bj8cv / (accessed on 22 October 2021). [CrossRef]

74. Weinbaum, D.; Veitas, V. Open ended intelligence: The individuation of intelligent agents. J. Exp. Theor. Artif. Intell. 2017, 29, 371-396. [CrossRef]

75. Wheeler, M. The Extended Mind Hypothesis; McDonald Institute for Archaeological Research Publications: Cambridge, UK, 2012.

76. Guo, K.; Yolles, M.I.; Fink, G.; Iles, P.A. The Changing Organisation: An Agency Approach; Cambridge University Press: Cambridge, UK, 2016.

77. Piaget, J. The Psychology of Intelligence; Harcourt and Brace: New York, NY, USA, 1950.

78. Uz, I. The Index of Cultural Tightness and Looseness Among 68 Countries. J. Cross-Cult. Psychol. 2014, 46, 319-335. [CrossRef]

79. Schmeichel, B.J.; Baumeister, R.F. Self-Regulatory Strength. In Handbook of Self-Regulation: Research, Theory, and Applications; Guilford Press: London, UK, 2004.

80. Odor, H. Organisational Culture and Dynamics. Glob. J. Manag. Bus. Res. 2018, 18, 23-29.

81. Mueller, E.T.; Dyer, M.G. Towards a Computational Theory of Human Daydreaming. In Proceedings of the Seventh Annual Conference of the Cognitive Science Society, Irvine, CA, USA, 15-17 August 1985.

82. Yolles, M. Agency, Generic ecosystems and sustainable development: Part 2 agency as an ecosystem. Kybernetes 2021. [CrossRef]

83. Vickers, G. Value Systems and Social Process; Routledge: London, UK, 2013.

84. Stowell, F. The Appreciative Inquiry Method-A Suitable Candidate for Action Research? Syst. Res. Behav. Sci. 2012, 30, 15-30. [CrossRef]

85. Alexiou, A. Understanding Multi-Agent Design as Coordination. Ph.D. Thesis, University of London, London, UK, 2007.

86. Birch, J. Natural selection and the maximization of fitness. Biol. Rev. 2015, 91, 712-727. [CrossRef]

87. Williams, A. A Model for General Collective Intelligence. arXiv 2020, arXiv:africarxiv / 6u984. Available online: https:/ / osf.io/ preprints / africarxiv/6u984 (accessed on 22 October 2021).

88. Foerster, V.H. Understanding: Essays on Cybernetics and Cognition; Spinger: New York, NY, USA, 2003.

89. Rocha, L. Eigenbehavior and symbols. Syst. Res. 1996, 13, 371-384. [CrossRef]

90. Varela, F. A calculus for self-reference. Int. J. Gen. Syst. 1975, 2, 5-24.

91. Spencer Brown, G. Laws of Form; The Julian Press: New York, NY, USA, 1969.

92. Reichel, A. Snakes all the Way Down: Varela's Calculus for Self-Reference and the Praxis of Paradise. Syst. Res. Behav. Sci. 2011, 28, 646-662. [CrossRef]

93. Kauffman, L. Laws of form and form dynamics. Cybern. Hum. Knowing 2002, 9, 49-63.

94. Kauffman, L. Eigenforms-Objects as tokens for eigenbehaviors. Cybern. Hum. Knowing 2003, 10, 73-90.

95. Kauffman, L. Reflexivity and Eigenform: The Shape of Process. Constr. Found. 2009, 4, 3.

96. Kauffman, L. Eigenforms and Quantum Physics. arXiv 2011, arXiv:1109.1892.

97. Dubois, D.M. Mathematical Foundations of Discrete and Functional Systems with Strong and Weak Anticipations. LNAI 2003, 2684, 110-132. [CrossRef]

98. Fisher, R. On the mathematical foundations of theoretical statistics. In Philosophical Transactions of the Royal Society A: Mathematical, Physical and Engineering Sciences; Royal Society: London, UK, 1922.

99. Frieden, B.R.; Binder, P.M. Physics from Fisher Information: A Unification. Am. J. Phys. 2000, 68, 1064-1065. [CrossRef]

100. Frieden, B. Science from Fisher Information; Cambridge University Press: Cambridge, UK, 2004.

101. Frieden, B.R.; Hawkins, R.J. Asymmetric information and economics. Phys. Stat. Mech. Appl. 2010, 389, 287-295. [CrossRef]

102. Karunanithi, A.T.; Cabezas, H.; Frieden, B.R.; Pawlowski, C.W. Detection and Assessment of Ecosystem Regime Shifts from Fisher Information. Ecol. Soc. 2008, 13, art22. [CrossRef] 
103. Karunanithi, A.T.; Garmestani, A.S.; Eason, T.; Cabezas, H. The characterization of socio-political instability, development and sustainability with Fisher information. Glob. Environ. Chang. 2011, 21, 77-84. [CrossRef]

104. Frieden, B. A Cooperative Universe-Beyond the Participatory. 2013. Available online: https://wp.optics.arizona.edu/rfrieden/ fisher-information/section-7/ (accessed on 17 August 2021).

105. Gatenby, R.; Frieden, B.R. Application of information theory and extreme physical information to carcinogenesis. Cancer Res. 2002, 62, 3675-3684.

106. Mutalik, P. How to Triumph and Cooperate in Game Theory and Evolution. QuantaMagazine. 9 November 2017. Available online: https: / / www.quantamagazine.org/how-to-triumph-and-cooperate-in-game-theory-and-evolution-20171109 (accessed on 20 July 2021).

107. Husserl, E. Addendum XXIII of The Crisis of European Sciences and Transcendental Phenomenology. J. Br. Soc. Phenomenol. 2013, 44, 6-9. [CrossRef]

108. Krippendorff, K. Content Analysis: An Introduction to its Methodology; Sage: New York, NY, USA, 2012.

109. Schwarz, E. Autogenesis. Available online: https://papers.ssrn.com/sol3/papers.cfm?abstract_id=3826203 (accessed on 22 October 2021).

110. Clark, A.; Chalmers, D. The extended mind. Analysis 1998, 58, 7-19. [CrossRef]

111. Duff, A.J.; Tahbaz, A.; Chan, C. The Interactive Effect of Cultural Intelligence and Openness on Task Performance. Res. Pract. Hum. Resour. Manag. 2012, 20,1-12.

112. Yolles, M.; Fink, G. Modelling mindsets of an agency. J. Organ. Transform. Soc. Chang. 2014, 11, 69-88. [CrossRef]

113. Thorndike, E.L. Intelligence and its uses. Harper's Mag. 1920, 140, 227-235.

114. Piaget, J. Development and learning. In Piaget Rediscovered; Cornell University Press: Ithaca, NY, USA, 1964; pp. 7-20.

115. Yolles, M. Towards a general hybrid theory in wicked problem structuring part 1: The foundation. Kybernetes 2020, 50, 467-489. [CrossRef]

116. Burns, N.; Grove, S.K. The Practice of Nursing Research: Appraisal Synthesis, and Generation of Evidence; Elsevier: Amsterdam, The Netherlands, 2009.

117. Yolles, M. Management Systems: A Viable Approach; Financial Times Pitman: London, UK, 1999.

118. Singh, S. Understanding the Bias-Variance Tradeoff, Towards Data Science. 2018. Available online: https://towardsdatascience. com/understanding-the-bias-variance-tradeoff-165e6942b229 (accessed on 19 June 2021).

119. David, O. Dynamic causal models and autopoietic systems. Biol. Res. 2007, 40, 487-502. [CrossRef] [PubMed]

120. Cartwright, N. What is this thing called efficacy? In Philosophy of the Social Sciences: Philosophical Theory and Scientific Practice; Cambridge University Press: Cambridge, UK, 2009; pp. 185-206.

121. DiMaggio, P. Culture and cognition. Annu. Rev. Sociol. 1997, 23, 263-287. [CrossRef]

122. Das, M.M.; Chithralekha, T.; Sathya, S. Static Context Model for Context Aware E-learning. Int. J. Eng. Sci. Technol. 2010, 2, 2337-2346.

123. Svobodová, A.; Koudelková, P. Collective intelligence and knowledge management as a tool for innovations. Econ. Manag. 2011, 16, 942-946.

124. Ringer, M. Leadership for collective thinking in the work place. Team Perform. Manag. Int. J. 2007, 13, 30-144. [CrossRef] 